\title{
RADIAL DEPENDENCE OF THE PATTERN SPEED OF M51
}

\author{
Sharon E. Meidt and Richard J. Rand \\ Department of Physics and Astronomy, University of New Mexico, 800 Yale Boulevard Northeast, Albuquerque, NM 87131 \\ Michael R. MERRIFIELD \\ School of Physics and Astronomy, University of Nottingham, University Park, Nottingham, NG7 2RD, UK \\ RAHUl SHETTY \\ Harvard-Smithsonian Center for Astrophysics, 60 Garden Street, Cambridge, MA 02138; and Initiative for Innovative Computing, \\ Harvard University, 60 Oxford Street, Cambridge, MA 02138 \\ AND \\ Stuart N. Vogel \\ Department of Astronomy, University of Maryland, College Park, MD 20742-2421 \\ Received 2008 May 2; accepted 2008 July 2
}

\begin{abstract}
The grand-design spiral galaxy M51 has long been a crucial target for theories of spiral structure. Studies of this iconic spiral can address the question of whether strong spiral structure is transient (e.g., interaction-driven) or longlasting. As a clue to the origin of the structure in M51, we investigate evidence for radial variation in the spiral pattern speed using the radial Tremaine-Weinberg (TWR) method. We implement the method on CO observations tracing the ISM-dominant molecular component. Results from the method's numerical implementation - combined with regularization, which smooths intrinsically noisy solutions - indicate two distinct patterns speeds inside $4 \mathrm{kpc}$ at our derived major axis P.A. $=170^{\circ}$, both ending at corotation and both significantly higher than the conventionally adopted global value. Inspection of the rotation curve suggests that the pattern speed interior to $2 \mathrm{kpc}$ lacks an ILR, consistent with the leading structure seen in $H S T$ near-IR observations. We also find tentative evidence for a lower pattern speed between 4 and $5.3 \mathrm{kpc}$ measured by extending the regularized zone. As with the original TW method, uncertainty in major axis position angle (P.A.) is the largest source of error in the calculation; in this study, where $\delta_{\text {P.A. }}= \pm 5^{\circ}$, a $\sim 20 \%$ error is introduced to the parameters of the speeds at P.A. $=170^{\circ}$. Accessory to this standard uncertainty, solutions with P.A. $=175^{\circ}$ (also admitted by the data) exhibit only one pattern speed inside $4 \mathrm{kpc}$, and we consider this circumstance under the semblance of a radially varying P.A.
\end{abstract}

Subject headings: galaxies: kinematics and dynamics — galaxies: spiral — galaxies: structure methods: numerical

Online material: color figures

\section{INTRODUCTION}

The large angular size and clear spiral structure of the nearly face-on spiral M51 make it ideal for studies of the nature and origin of grand design spiral structure. Two scenarios dominate the discussion in the literature, each based on opposing theories: strong spiral structure as a quasi-stationary density wave (e.g., Lin \& Shu 1964), or as a transient feature due to interaction with nearby companion NGC 5195 (e.g., Tully 1974).

Observations of both the stellar and gaseous components reveal consistencies with the density wave interpretation at some level (see Elmegreen et al. 1989; Vogel et al. 1993; Rand 1993). In accord with the seminal study of Tully (1974), which attributes the (transient) outer pattern to the interaction with its companion and proposes that the inner arms are likely spiral density waves also driven by the encounter, Elmegreen et al. (1989) and Vogel et al. (1993) find independent evidence for two different pattern speeds. If the strong spiral's corotation radius overlaps with the ILR of the outer, material pattern, as is suspected (e.g., Tully 1974; Elmegreen et al. 1989), this may indicate the stimulation of an inner spiral wave mode by the outer material spiral via mode-coupling (Tagger et al. 1987). But observationally it remains unclear whether, if driven by the outer pattern, the strong spiral structure is transient, or has survived a few rotation times (e.g., as speculated by Vogel et al. 1993).
In the simulations of both Salo \& Laurikainen (2000a) and Howard \& Byrd (1990) structure throughout the disk is well reproduced by multiple passages of the (bound) orbiting companion, and the nuclear structure, in particular, seems intimately related to the inward propagation of multiple tidally induced perturbations (Salo \& Laurikainen 2000b). Pursuant to the study of Toomre \& Toomre (1972) such simulations of M51 have proved indispensable for exploring and motivating scenarios in favor of short-lived waves. In the short-lived wave paradigm, propagating wave packets evolved from kinematic distortions in the outer disk may be swing-amplified (Toomre 1981), causing a strong response in the inner disk. As predicted by Salo \& Laurikainen (2000b) the wave speed has a complex radial dependence, featuring a constant pattern speed for the dominant $m=2$ structure out to $1.2-1.8 \mathrm{kpc}$ (depending on the disk mass assumed), followed by a superposition of structures described by a pattern speed that decreases with radius, down to $\sim 10-20 \mathrm{~km} \mathrm{~s}^{-1} \mathrm{kpc}^{-1}$ by $\sim 4.6 \mathrm{kpc}$.

Knowledge of the pattern speed of the structure can, in principle, both distinguish between and reconcile the short- and longlived wave scenarios, and so many studies have focused on measuring and characterizing this parameter (see, e.g., Elmegreen et al. 1989; Tully 1974; Salo \& Laurikainen 2000b; GarciaBurillo et al. 1993). The pattern speed of the outer spiral has long been proposed near $10-20 \mathrm{~km} \mathrm{~s}^{-1} \mathrm{kpc}^{-1}$. In the inner disk, 
application of the traditional, model-independent method of Tremaine \& Weinberg (1984, hereafter TW) using CO observations yields a pattern speed $\Omega_{p}=38 \mathrm{~km} \mathrm{~s}^{-1} \mathrm{kpc}^{-1}$ (Zimmer et al. $2004)$, in general agreement with the determinations based on resonance locations of Elmegreen et al. (1989) and Tully (1974) (but higher than the pattern speed $\Omega_{p}=27 \mathrm{~km} \mathrm{~s}^{-1} \mathrm{kpc}^{-1}$ found by Garcia-Burillo et al. 1993). Although the TW analysis shows evidence for significant departures from the expected relation for a constant pattern speed in both the inner- and outermost regions of the disk, the method cannot quantitatively account for any suspected radial variation of the pattern speed.

The radial TW (hereafter TWR) method (Merrifield et al. 2006; Meidt et al. 2008) should prove an invaluable resource in this regard, since with it we can characterize the angular speeds of distinct patterns and their possible radial variation. For the first time, we are able to observationally address issues related to the complex nature and persistence of spiral patterns and the connection, if any, between multiple pattern speeds in a single disk.

Like its traditional counterpart, the TWR method, summarized in $\S 2$, relies on the use of a kinematic tracer found to obey continuity. Here, we consider the ISM-dominant molecular component in the inner disk of M51 as traced by CO observations. In $\S \S 3.1$ and 3.2 we describe these observations and review the arguments which establish their conformity with the assumptions of the method. In $\S 3.3$ we formulate the TWR quadrature and motivate the models developed for testing.

Results of the regularized TWR calculation applied to the inner disk are presented in $\S 4.2$. There we establish a best estimate for the pattern speed(s) of the bright spiral structure by considering the characteristics of solutions over a $\pm 5^{\circ}$ range of disk position angles (P.A.s) (§ 4.3); according to the findings of Debattista (2003) we can expect uncertainty in the P.A. to be the dominant source of systematic error in the calculation. We also compare this estimate to other tested models of the radial dependence in $\S 4.4$.

In an effort to authenticate the TWR estimate, in $\S 4.5$ we relate our measurements to other independent evidence for more than a single pattern speed in the inner disk of M51 and investigate the resonance locations and overlaps that they entail. We also consider our measurements in light of relevant findings throughout the literature, including those of Shetty et al. (2007), in $\S 4.6$, and Henry et al. (2003, in $\S 4.7)$. Final results are summarized in a conclusion section.

\section{THE TWR METHOD WITH REGULARIZATION}

The radial modification of the TW method (Merrifield et al. 2006; Meidt et al. 2008, hereafter M08) delivers a derivation for radially dependent pattern speeds measurable from observationally accessible quantities. The so-called TWR calculation proceeds under assumptions parallel to those of the original method, namely, that the disk of the galaxy is flat (unwarped); that the surface density of a disk component, which must obey continuity, becomes negligibly small at some radius and all azimuths within the map boundary (thereby critically yielding converged integrals; see below); and that the relation between the emission from this component and its surface density is linear, or if not, suspected deviations from linearity can be modeled.

Departure from the traditional method (which assumes that the disk contains a single, well-defined rigidly rotating pattern) emerges by allowing that $\Omega_{p}=\Omega_{p}(r)$ - and the surface density of the tracer $\Sigma(x, y, t)=\Sigma\left(r, \phi-\Omega_{p}(r) t\right)$. Integration of the continuity equa- tion obeyed by the tracer thereupon yields a Volterra integral equation of the first kind for $\Omega_{p}(r)$,

$$
\int_{r=y}^{\infty}\left\{\left[\Sigma\left(x^{\prime}, y\right)-\Sigma\left(-x^{\prime}, y\right)\right] r\right\} \Omega_{p}(r) d r=\int_{-\infty}^{\infty} \Sigma v_{y} d x
$$

where $x^{\prime}(r, y)=\left(r^{2}-y^{2}\right)^{1 / 2}$ (Merrifield et al. 2006). This equation can be cast in terms of $x_{\text {obs }}$ and $y_{\text {obs }}$, the coordinates in the plane of the sky along the major and minor axes, respectively, and $v_{\text {obs }}$, the observed line-of-sight velocity, since for a galaxy projected onto the sky plane with inclination $\alpha, x=x_{\mathrm{obs}}, y=$ $y_{\text {obs }} / \cos \alpha$, and $v_{y}=v_{\text {obs }} / \sin \alpha$.

When the integral on the left of equation (1) is replaced with a discrete quadrature for different values of $y=y_{i}$ and $r=r_{j}$ (represented in Fig. 1 of M08), equation (1) takes the form of the matrix expression

$$
K_{i j} \Omega_{j}=b_{i}
$$

with $\boldsymbol{K}$ an upper triangular $N \times N$ square matrix. This can be solved numerically for a total of two independent measures of $\Omega_{p}(r)$, one from either side of the galaxy $(y>0$ and $y<0)$.

As described by Merrifield et al. (2006) (and depicted in Fig. 2 of M08), solving equation (2) by standard back-substitution results in the propagation of errors from large radii inward, whereby solutions inescapably display noisy oscillations. As demonstrated there, applied first to Sb galaxy NGC 1068, this effect can be impeded most simply by adopting a relatively large bin width; the TWR solution in this case is found to decrease with radius, and yield a winding time estimate for the two-armed structure.

But in general, as found in application to simulations (M08), smaller radial bins are preferable to ensure accurate assessment of radial variation. In addition, noisy behavior in solutions tends to be amplified when the quadrature extends out to the edge of the surface brightness (a requirement argued for by M08), which not least imposes that the outermost bins generally cover the lowest $\mathrm{S} / \mathrm{N}$ regions in the disk. Combined with a relatively small bin, numerical solutions as a result of inward error propagation display a systematic offset in each bin between measurement and the actual value, preventing accurate determination of $\Omega_{p}(r)$ (M08).

As shown in M08, regularization provides an effective means of reducing the intrinsic propagation of noise in solutions while maintaining the precision required to accurately identify true radial variation. There, regularized TWR calculations were applied successfully to simulated disks featuring multiple pattern speeds in distinct radial zones as well as spiral winding.

Following M08, then, we introduce a regularizing operator, or smoothing functional $S$, containing a priori information in the manner of Tikhonov-Miller regularization (Tikhonov \& Arsenin 1997; Miller 1970) into the $\chi^{2}$ estimator minimized by solutions $\Omega_{j}$ of equation (2), whereby minimization returns smoothed solutions according to (in matrix form)

$$
\left(\overline{\boldsymbol{K}}^{T} \cdot \overline{\boldsymbol{K}}+\lambda \boldsymbol{S}\right) \cdot \Omega=\overline{\boldsymbol{K}}^{T} \cdot \bar{b}
$$

where the elements of $\overline{\boldsymbol{K}}$ and $\overline{\boldsymbol{b}}$ are $K_{i j} / \sigma_{i}$ and $b_{i} / \sigma_{i}$, respectively (with errors $\sigma_{i}$ representing the measurement error of the $i$ th data point $b_{i}$ ), and the parameter $\lambda$ controls the degree of smoothness achieved in solutions. Details for the full calculation and analysis can be found in M08; we proceed by highlighting only a few of the main precepts.

By incorporating simple expectations from theory and observation into the smoothing $S$, the solution of equation (3) yields 
smoothed, testable models for $\Omega_{p}(r)$. These models we restrict to simple forms and consider only polynomial solutions with constant, linear, and quadratic radial dependence. (The elements of the smoothing $\boldsymbol{S}$ are associated with the minimization of the $n$th derivative of $\Omega(r)$ for each polynomial solution of order $n$.) These polynomial models can be incorporated into step functions which parameterize the radial domains of multiple pattern speeds (see M08).

The best-fit global solution constructed from the average of like-model solutions from the two sides is established using the standard $\chi_{\nu}^{2}\left(\chi^{2}\right.$ per degree of freedom) statistic, as in M08. (Note that an explicit assumption here is that all patterns in the disk are indeed global.) To summarize, once equation (3) is solved with a set of prescribed smoothings on each side, we use equation (2) to generate a complete set of $\langle v\rangle_{i}=b_{i} /\left(\int \Sigma d x\right)_{i}$ for each global model. The $\chi_{\nu}^{2}$ fit of the model - reproduced to actual $\langle v\rangle_{i}$ given global measurement error $\sigma^{\langle v\rangle}$ (defined as the average of the individual errors $\sigma_{i}^{\langle v\rangle}$ for each slice) - is then calculated for each. For this $\chi_{\nu}^{2}$, we adopt the uniform weighting scheme advocated by $\mathrm{M} 08$.

According to the M08 prescription, where measurement errors for each slice reflect random noise in the data, for this analysis we assign errors $\sigma_{i}^{\langle v\rangle}$ that define the change in the measured $\langle v\rangle$ introduced by a change in the chosen flux cutoff in the first moment map. Specifically, the error in the $\langle v\rangle$ measured from a map with an $n \sigma$ level cutoff is defined as

$\sigma^{\langle v\rangle}=\frac{1}{\sqrt{2}}\left[\left(\langle v\rangle_{(n-1) \sigma}-\langle v\rangle_{n \sigma}\right)^{2}+\left(\langle v\rangle_{(n+1) \sigma}-\langle v\rangle_{n \sigma}\right)^{2}\right]^{1 / 2}$

for each slice $i$. The average of the individual errors then define the global measurement error across the entire disk where $\sigma^{\langle v\rangle}=\left(\sum_{i=1}^{2 N} \sigma_{i}^{\langle v\rangle}\right) / 2 N$ (and $N$ is the number of bins/slices used in the TWR calculation on a single side). [Note that this error relates to the error $\sigma_{i}$ for each slice in eq. (3) through $\left(\int \Sigma d x\right)_{i}$.

Although these random errors are used in the goodness-of-fit criterion, the overall error in the measurement of $\Omega_{p}(r)$ given by the best-fit global model solution is defined relative to systematic errors in the calculation. Uncertainty in the assumed P.A., for example, has the largest potential for introducing errors into $\langle v\rangle_{i}$, or conversely, the $b_{i}$ in equation (3), and is the dominant source of error in TW calculations (Debattista 2003; M08). We assess this error by testing the sensitivity of the solutions to departures from the nominal value for the P.A. (or inclination, for instance). Unless otherwise specified, in this paper all reported error bars reflect the influence of P.A. uncertainty alone. As for inclination errors, apart from the change introduced in the pattern speed measurements through a change in $\sin \alpha$, these prove to be of little additional consequence to the accurate placement of radial bins defined in the quadrature (as suggested by M08), despite the relatively low inclination (we adopt $\alpha=24^{\circ}$; see Table 1) of the disk of M51. We therefore do not report this error, but instead note that a change in the inclination by $\delta_{\alpha}= \pm 3^{\circ}$ corresponds to a fractional variation of about $12 \%$ in the pattern speed estimates reported here.

\section{APPLICATION TO M51}

\subsection{Observations}

In this paper we consider the disk of M51 traced by highresolution $\mathrm{CO}$ observations. As described in its initial publication (Shetty et al. 2007), the cube consists of the BIMA Survey of Nearby Galaxies (SONG) observation together with several additional pointings which extend the map out to $r \sim 280^{\prime \prime}$ and provide higher angular resolution in the central regions (see the
TABLE 1

Parameters Used in the TWR Calculation

\begin{tabular}{|c|c|}
\hline Parameter & Value \\
\hline 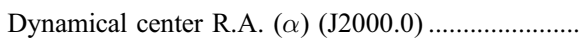 & $13^{\mathrm{h}} 29^{\mathrm{m}} 52.71^{\mathrm{s}}$ \\
\hline 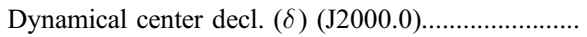 & $47^{\circ} 11^{\prime} 42.80^{\prime \prime}$ \\
\hline 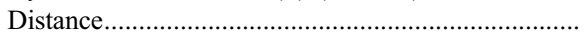 & $9.5 \mathrm{Mpc}$ \\
\hline 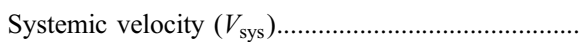 & $469 \mathrm{~km} \mathrm{~s}^{-1}$ (LSR) \\
\hline Position angle & $170^{\circ} \pm 5^{\circ}$ \\
\hline Inclination ............... & $24^{\circ} \pm 3^{\circ}$ \\
\hline
\end{tabular}

NotE.-The dynamical center and inclination angle are adopted from Shetty et al. (2007). Entries for $V_{\text {sys }}$ and P.A. originate from the tilted ring analysis of the first moment of the $\mathrm{CO}$ cube using the GIPSY task ROTCUR.

beginning of $\S 3.3)$. A complete description of the data can be found in Shetty et al. (2007). The $2 \sigma$ zeroth- and first-moment maps used in this analysis, derived from the full cube, are shown in Figure 1. Measurement errors given by uncertainty in the flux cutoff are defined relative to maps at the 1 and $3 \sigma$ levels.

\subsection{Establishing Molecular Dominance}

The measured intensities and velocities in Figure 1 are suitable for use with the TWR method provided that the assumptions listed in the previous section are satisfied. While the continuity requirement can be particularly limiting, Zimmer et al. (2004) and Rand \& Wallin (2004) argue that CO emission, the standard tracer of the molecular component of the ISM, suitably meets the TW assumptions for galaxies where the ISM is everywhere dominated by molecular gas. This is founded on the low true efficiency of star formation in spirals, which implies that only a small fraction of molecular gas is converted into stars on orbital timescales, while molecular dominance implies that the conversion of molecular hydrogen into other phases of the ISM occurs at low levels.

Zimmer et al. (2004) applied the TW method under this premise, showing with $\mathrm{CO}$ and $\mathrm{H}$ I observations that the gas content of M51 is in fact dominated by molecular hydrogen where $\mathrm{CO}$ is detected. The CO observations used in this work can be similarly asserted to obey continuity: assuming a conversion factor between $\mathrm{CO}$ intensity and $\mathrm{H}_{2}$ column density $X=2 \times 10^{20} \mathrm{~cm}^{-2}$ $\left(\mathrm{K} \mathrm{km} \mathrm{s}^{-1}\right)^{-1}$, molecular hydrogen is found dominant over the majority of the CO-emitting disk (roughly $R<105^{\prime \prime}$ ), where $N\left(\mathrm{H}_{2}\right) / N(\mathrm{HI}) \sim 10$ (Shetty et al. 2007).

The possibility of variation in the $\mathrm{CO}-\mathrm{H}_{2}$ conversion factor has also been addressed by Zimmer et al. (2004), who find in a series of tests applied to M51 that neither a linear relationship between metallicity and $X$-factor nor arm-interarm variations at levels suggested by Garcia-Burillo et al. (1993) produce a significant change in the derived pattern speed estimate.

The negligible effect of radial dependence in $X$ can be largely attributed to the cancellation of axisymmetry with TW integration along each slice (see Zimmer et al. 2004 for a complete account). Analogously, for the TWR method, as long as the metallicity changes negligibly over the width of a radial bin, we can expect little change in the results of calculations that assume an approximately constant $X$-factor throughout the disk. We have confirmed this to be the case here; modeled according to the Bresolin et al. (2004) metallicity gradient $0.02 \mathrm{dex} \mathrm{kpc}^{-1}$, an increase in $X$ with radius produces negligible change in the measured solutions.

An arm-interarm contrast as suggested by Garcia-Burillo et al. (1993), on the other hand, in general may not so readily translate from TW to TWR calculations inconsequentially. Currently, assessing the particular effect of azimuthal variation in the $X$ factor on the results of the TWR method is beyond the scope of this 

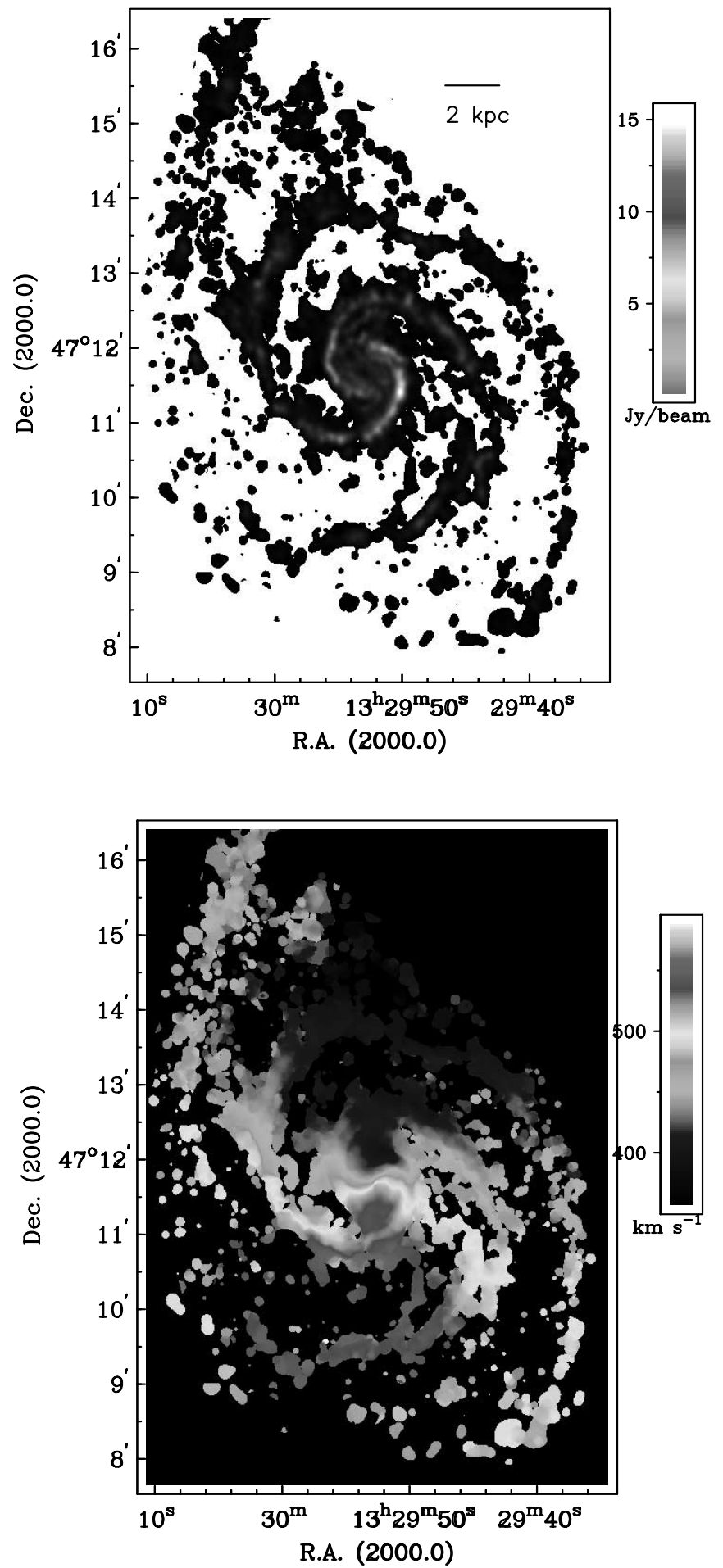

FIG. 1.-Zeroth (right) and first (left) moment maps of the M51 CO cube (originally presented in Shetty et al. 2007). The horizontal bar in the top right corner indicates the physical scale. The $y>0$ quadrature generally covers the eastern half of the image (depending on the value of the P.A. adopted), while the $y<0$ quadrature covers the western. [See the electronic edition of the Journal for a color version of this figure.]

work. Here, we rely on the results of Zimmer et al. (2004) to assert that, to a first approximation, variation in $X$ should not compromise the analysis as presented here.

\subsection{Defining the Quadrature and Developing Testable Models}

In order to achieve as accurate a quadrature as possible and also limit errors caused by the misdesignation of any transitions (e.g., given the finite bin width to which solutions are confined; M08), we adopt a radial bin width $\Delta r=0.23 \mathrm{kpc}(D=9.5 \mathrm{Mpc})$. This corresponds to the limiting resolution $\left(\sim 4^{\prime \prime}\right)$ of the map at the innermost radii. Since with the majority of our analysis of M51 we are most interested in characterizing the pattern speeds of the bright spiral structure, this choice is expected to yield high-quality solutions for the pattern speeds in this region in particular.

At the largest radii (and in interarm regions) the resolution decreases to $6^{\prime \prime}-13^{\prime \prime}$ (Shetty et al. 2007). Although in principle the quadrature can accommodate a nonuniform bin width, we maintain $\Delta r=0.23 \mathrm{kpc}$ throughout the disk and rely instead on the allocation of information administered by regularization. We assert that, even with our $4.5^{\prime \prime}$ radial bins, regularized TWR calculations are prevented from oversampling the data as long as any distinct regions parameterized by the models are larger than the resolution.

As assessed in M08, we can expect departures from the nominal values of the parameters appearing in equation (2) to introduce nonnegligible errors into the TWR solutions. Uncertainty in the major axis P.A. is the dominant source of systematic error in the calculation, resulting in errors on the order of $20 \%$ in TWR pattern speeds (M08). Since the kinematic parameters of M51 are notoriously difficult to constrain, perhaps the greatest challenge to the accuracy of our solutions lies in the accuracy with which we can constrain the quadrature.

To best equip the analysis in this capacity, then, we survey both our own derivations of the kinematic parameters and those from the literature. For the coordinates of the center of rotation and the disk inclination angle, for example, we rely on the values from the study of Shetty et al. (2007). These we then adopt in fits of a tilted ring model to the $\mathrm{CO}$ velocity field with the Groningen Image Processing System (GIPSY) program ROTCUR to determine the systemic velocity and the kinematic line of nodes (as well as the rotation velocity as a function of radius). The resulting parameters (listed in Table 1) are consistent with most previous determinations. Note, however, that rather than adopting the range of P.A.s $\left(170^{\circ}-180^{\circ}\right)$ considered by Shetty et al. (2007), we initially choose P.A. $=170^{\circ} \pm 5^{\circ}$. This is principally in order that our results are more easily compared with the majority of studies on M51, especially those which entail estimates for the pattern speed. In addition, for this study we assume $D=9.5 \mathrm{Mpc}$; with the alternative $D=7.7 \mathrm{Mpc}$ (more common to recent studies), all distances reported here decrease by a factor of $\sim 0.2$, while all pattern speeds reciprocally increase.

According to the arguments in M08, we extend the unique quadrature established with the values in Table 1 out to the map boundary $\pm y_{\max }$ in order to ensure that all information critical for characterizing the patterns of interest is accounted for. Since the emission extends (roughly east-west) out to $\pm y_{\max }=145^{\prime \prime}$, this defines the maximum radial extent of the quadrature $R_{\max }=$ $y_{\max } / \cos \alpha=7.3 \mathrm{kpc}$, and hence the limit of integration along each slice $\pm X_{i}=\left(R_{\max }-y_{i} / \cos \alpha\right)^{1 / 2}$. Although this does place $X_{i}$ within the edge of the emission at small $|y|$ (given the elongated emission in this map from north to south and the low disk inclination), the radial range of the quadrature is still comfortably outside the radius where the integrals converge.

As diagnosed by M08, with this fairly extensive quadrature solutions $\Omega_{p}(r)$ are at risk of regularization-induced bias. This bias is defined for the particular case when bins cover a region that displays only faint emission, has little information from a strong pattern, or is suspected of sustaining multiple patterns; when these bins are prescribed an unrealistic model, the accuracy of the remainder of the solution can be jeopardized. 
Evidence for regions in the outer disk of M51 susceptible to regularization-induced bias are identifiable a priori in the intensity map and its Fourier power spectrum. Later, in $\S 4.2$, where we address this bias and its signatures, we adopt the countermeasure developed by M08, wherein the compromised bins are calculated without regularization. This imposes the additional parameterization of a cut radius $r_{c}$ on our model solutions, interior to which regularization proceeds as defined in equation (3). (See M08 for a description of the calculation and analysis in this case.) In practice, we unregularize only as long as we can ensure the sustained effectiveness of regularization in the rest of the calculation, given that an increased number of unregularized bins promotes the reintroduction of unamendable propagating noise.

In order to test for the possibility of multiple pattern speeds and/or winding, models additionally parameterize either single or multiple distinct radial domains over which the solution can vary as zeroth-, first-, or second-order polynomials. Although in general we test all possible models at each stage of the analysis, in some cases we restrict our consideration to only those polynomials for which the degree of freedom plus 3-4 bins does not exceed the number of bins in a given domain.

\section{M51: RESULTS}

\subsection{Isolating the Inner Structure Pattern Speed}

As in previous applications of the method, we make use of a priori information to develop physically motivated models for $\Omega_{p}(r)$. And as with all such models, in order that they supply rigorous estimates we must also account for evidence suggesting susceptibility to regularization-induced bias. In the surface brightness (Fig. 1) and its Fourier decomposition (Fig. 2) we identify a region outside $r \sim 4 \mathrm{kpc}$, in particular, where both the surface brightness and power in the $m=2$ component are low, an indication that the information to be extracted there is potentially unreliable and difficult to constrain through modeling.

This is manifest in solutions for which regularization is employed throughout the full extent of the emission. Bins at large radii in the lowest $\chi^{2}$ solutions exhibit a significant degree of variation in their modeling, confirming that constraining the outer pattern speed is difficult. According to the conclusion drawn by M08 in tests of the regularized TWR calculation on simulations, this challenges the accuracy with which all inner bins can realize the true pattern speed. We therefore initially consider models which parameterize a cut radius $r_{c}=4.1 \mathrm{kpc}$, beyond which all bins are calculated without regularization. In testing, we find this cut radius to coincide with a clear minimum in the $\chi^{2}$, with all other best-fit parameters held fixed.

A second, shallower minimum at $r_{c}=5.3 \mathrm{kpc}$ is also compelling, and we consider its parameterization in models of $\Omega_{p}(r)$ in the analysis that follows, as well. This location may well be reasonable for the separation of the patterns given that it seems to match expectations for the location where the outer, material pattern begins. We cite in particular the study of Elmegreen et al. (1989), who, like Tully (1974) argue that OLR occurs at the termination of the bright, inner spiral structure and in the pretext of mode-coupling therein identify an overlap between the CR of the inner pattern with the ILR of a $10-20 \mathrm{~km} \mathrm{~s}^{-1} \mathrm{kpc}^{-1}$ outer pattern (e.g., that first proposed for the material pattern by Tully 1974). This places the innermost extent of the material pattern at $r \sim$ $6.0 \mathrm{kpc}$ (adopted into the distance convention used here). Vogel et al. (1993) also argue for a similar corotation radius based on observations of streaming motions in the ionized gas component of the ISM. And while this does not locate the inner extent of the outer pattern, it nevertheless implies that the outer arms are sep-

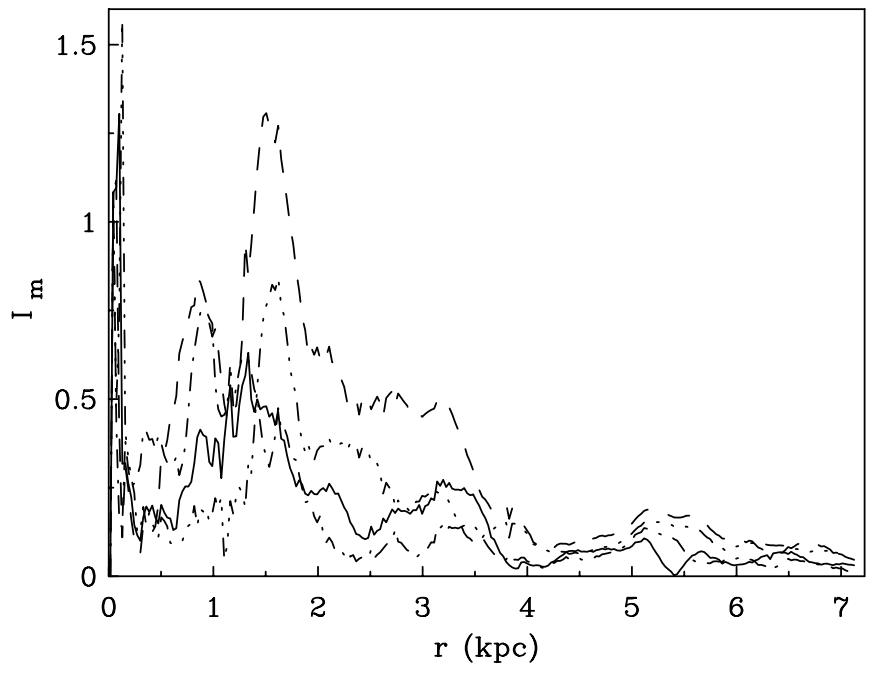

FIG. 2.- Fourier power spectrum of the moment zero map shown in Fig. 1. Modes up to $m=4$ are plotted as a function of radius for $m=1$ (dash-dotted line), $m=2$ (dashed line), $m=3$ (solid line), and $m=4$ (dash-double-dotted line). [See the electronic edition of the Journal for a color version of this figure.]

arate from, and have a lower pattern speed than, the inner arms (Vogel et al. 1993). Consequently, it is consistent with the conclusion of Elmegreen et al. (1989).

A transition from an inner to an outer pattern is also recognizable in the tidal perturbation-only model of Salo \& Laurikainen (2000b). There, an independent spiral pattern with corotation near $r=4.6 \mathrm{kpc}$ is found to be followed by structure at the lower $10-20 \mathrm{~km} \mathrm{~s}^{-1} \mathrm{kpc}^{-1}$ pattern speed. Any resonance overlap, however, they argue is likely coincidental since the value of the higher, inner speed is associated with the maximum in $\Omega-\kappa / 2$, while the lower speed is determined mainly by external forcing.

In addition, as revealed in the sections to follow, while results with $r_{c}=4.1 \mathrm{kpc}$ indicate much higher speeds $\left(\sim 50-100 \mathrm{~km} \mathrm{~s}^{-1}\right.$ $\left.\mathrm{kpc}^{-1}\right)$ than the TW method $\left(\Omega_{p}=38 \mathrm{~km} \mathrm{~s}^{-1} \mathrm{kpc}^{-1}\right)$, with $r_{c}=$ $5.3 \mathrm{kpc}$ solutions measure a much lower speed exterior to $r \simeq$ $4 \mathrm{kpc}$, at least qualitatively more consistent with the gross overall speed estimated with the TW calculation.

\subsection{Best-Fit Models}

When we minimize the influence of the suspected material pattern in the TWR solutions by calculating the bins in the outer zone without regularization, we in principle maximize the leverage on the inner structure. In doing so, we find the data at the nominal P.A. to be well fit by two distinct pattern speeds interior to $r_{c}=4.1 \mathrm{kpc}$. The overall pattern speed solution with P.A. uncertainty $\delta_{\text {P.A. }}= \pm 5^{\circ}$, to be discussed at length below, is represented in Figure 3. Following the treatment of M08 for constructing errors on the measurement from a particular observational scenario, error bars represent the dispersion of the parameters in the best-fit solution derived with a two-pattern speed model at P.A. = $165^{\circ}, 170^{\circ}$, and $175^{\circ}$. As will be discussed further in $\S 4.5$, these two pattern speeds both end at corotation, within the uncertainties.

To quantify the relative benefit of the two pattern speed solution, in Table 2 we list the $\chi_{\nu}^{2}$ estimate for several model solutions calculated over all slices in the TWR quadrature. In this table we also consider a $\chi_{\nu}^{2}$ over slices in the zones $0<|y|<2.3$ and $2.3<|y|<4.1 \mathrm{kpc}$ at the nominal P.A. We expect the $\langle v\rangle$ for slices in each of these zones to predominantly reflect measurements in the radial bins $r=|y|$, so these separated $\chi_{\nu}^{2}$ (labeled $\chi_{\nu, s}^{2}$ hereafter) should provide a fair comparison of $\Omega_{p}(r)$ from model to model at these radii. (Note, however, that all outer 


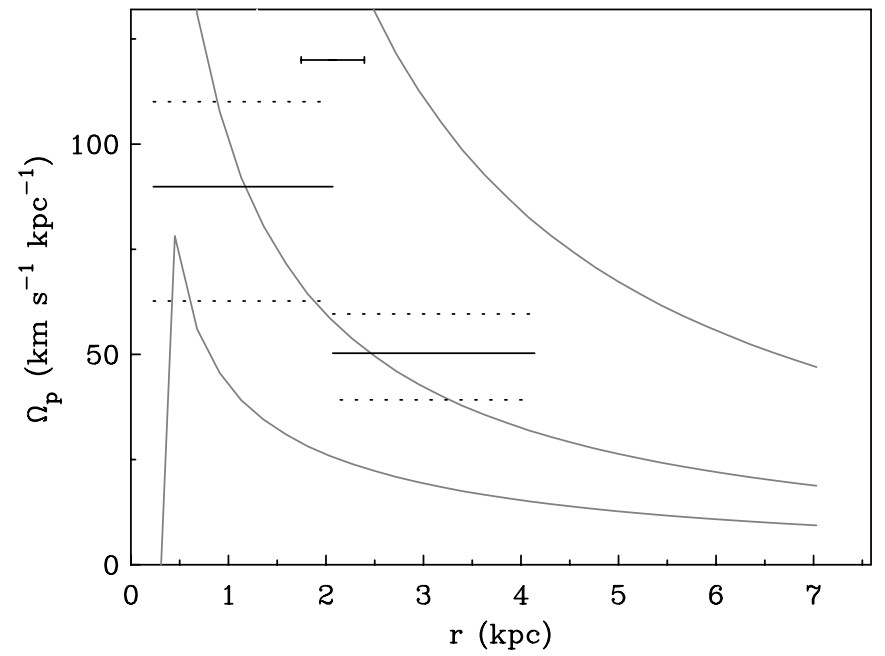

FIG. 3.-Best-fit regularized solution with $r_{c}=4.1 \mathrm{kpc}$ for P.A. $=170_{-55^{\circ}}^{\circ}$. For this solution, bins exterior to $r=4.1 \mathrm{kpc}$ (not shown) have been calculated without regularization. Dashed lines represent the difference from solutions derived with a two pattern speed model at P.A. $=165^{\circ}$ and $175^{\circ}$. Horizontal error bars represent the dispersion in $r_{t, 1}$ and $r_{t, 2}$ from P.A. to P.A. The values in the zone of the bright spiral structure correspond to $\Omega_{p, 1}=90_{+20}^{-27} \mathrm{~km} \mathrm{~s}^{-1} \mathrm{kpc}^{-1}$ out to $r_{t, 1}=2.1 \pm 0.3 \mathrm{kpc}$ and $\Omega_{p, 2}=50_{-11}^{+9} \mathrm{~km} \mathrm{~s}^{-1} \mathrm{kpc}^{-1}$ out to $r_{c}$. Curves for $\Omega$, $\Omega \pm \kappa / 2$, and $\Omega-\kappa / 4$ (see $\S 4.5)$ are shown in gray. [See the electronic edition of the Journal for a color version of this figure.]

bins also appear in the $\langle v\rangle$ reproduced by solutions in these zones.)

The $\chi_{\nu}^{2}$ fit over all slices principally suggests that the two pattern speed solution and the single, constant speed solution yield better agreement with the data than the quadratic solution. For the former two solutions, the $\chi_{\nu}^{2}$ values are nearly indistinguishable at this P.A. (see the last column in Table 2). From the $\chi_{\nu, s}^{2}$, on the other hand, it is clear that inside $r \simeq 2 \mathrm{kpc}$ the fit of the two pattern speed solution is significantly better than that of the constant speed.

Comparisons with the $\langle v\rangle$ reproduced by the best-fit two pattern speed model clearly demonstrate the incompatibility of the constant speed model, as shown on the left side of Figure 4. There, the $\langle v\rangle_{i}$ (top) and residuals (bottom) at each slice position reproduced by the best-fit solution are plotted along with those reproduced by the best polynomial solution with constant pattern speed $\Omega_{p}=55 \mathrm{~km} \mathrm{~s}^{-1} \mathrm{kpc}^{-1}$ calculated over the same radial zone. In the latter case, a greater departure from the measured values is readily apparent at slices inside $|y| \sim 2 \mathrm{kpc}$ as compared with the best-fit solution, which transitions from an outer speed of
$\Omega_{p}=50 \mathrm{~km} \mathrm{~s}^{-1} \mathrm{kpc}^{-1}$ to an inner speed of $\Omega_{p}=90 \mathrm{~km} \mathrm{~s}^{-1} \mathrm{kpc}^{-1}$ at $r_{t}=2.3 \mathrm{kpc}$. In fact, the constant solution fits the data better than the two-speed solution in only 2 of the 23 such slices.

In contrast, the quadratic solution with $r_{c}=4.1 \mathrm{kpc}$ at P.A. $=$ $170^{\circ}$, which declines smoothly with radius from $\sim 95 \mathrm{~km} \mathrm{~s}^{-1}$ $\mathrm{kpc}^{-1}$, grants nearly comparable agreement with the measured values that the two pattern speeds entail, over a number of slices. However, the fit of this solution weakens at slices between $|y| \sim$ 2.3-4 kpc (clear from the $\chi_{\nu, s}^{2}$ ), raising its $\chi_{\nu}^{2}$ well above that of the two-speed model. By comparison, then, it would seem that the two pattern speed solution presents the best fit for slices at both small and large radii (in slices $|y| \leq 4 \mathrm{kpc}$ ).

\subsubsection{Extended Models}

When the regularized zone $4.1<r<5.3 \mathrm{kpc}$ is included in solutions, the best-fit solution once again measures two pattern speeds inside $4 \mathrm{kpc}$, but now a third, distinct pattern speed is also parameterized. Figure 5 plots this best-fit solution, where, again, error bars are defined by the dispersion in the lowest $\chi_{\nu}^{2}$ solutions derived with a three pattern speed model at P.A. $=170^{\circ}, 165^{\circ}$, and $175^{\circ}$.

According to the $\chi_{\nu, s}^{2}$ in Table 2 for solutions with $r_{c}=5.3 \mathrm{kpc}$, in both inner and outer zones this best-fit solution is superior to a two-speed solution, which transitions from a single constant $62 \mathrm{~km} \mathrm{~s}^{-1} \mathrm{kpc}^{-1}$ pattern speed inside $3.2 \mathrm{kpc}$ to a lower $20 \mathrm{~km} \mathrm{~s}^{-1}$ $\mathrm{kpc}^{-1}$ speed, and also, once again, to either of the two polynomial solutions considered here.

The quadratic solution (decreasing from $180 \mathrm{~km} \mathrm{~s}^{-1} \mathrm{kpc}^{-1}$ ) fits relatively well in the innermost bins, but overall the fit is now less comparable to that provided by solutions measuring three pattern speeds. The agreement between the constant model solution-reproduced $\langle v\rangle_{2}$ and the data also weakens, relative to the best-fit solution, especially inside $r=4 \mathrm{kpc}$. This can be attributed to the decrease in the value measured with the constant model, from $\Omega_{p}=55 \mathrm{~km} \mathrm{~s}^{-1} \mathrm{kpc}^{-1}$ with $r_{c}=4.1 \mathrm{kpc}$ to $\Omega_{p}=$ $27 \mathrm{~km} \mathrm{~s}^{-1} \mathrm{kpc}^{-1}$ with $r_{c}=5.3 \mathrm{kpc}$. Incidentally, this is a clear indication that not only is the pattern speed in the zone $4 \lesssim r \lesssim$ $5 \mathrm{kpc}$ lower than $\Omega_{p}=55 \mathrm{~km} \mathrm{~s}^{-1} \mathrm{kpc}^{-1}$, but as such undeniably influences all bin values calculated inward with this type of model, thereby interfering with accurate measurement interior.

In contrast, when the zone $4 \lesssim r<5.3 \mathrm{kpc}$ is distinct and isolated, multispeed models are nearly free of such inaccuracy. In both the two- and three-speed solutions with $r_{c}=5.3 \mathrm{kpc}$, the inner pattern speeds are nearly identical to the values measured in solutions with $r_{c}=4.1 \mathrm{kpc}$. This seems to suggest that, despite

TABLE 2

$\chi^{2}$ Model Comparison

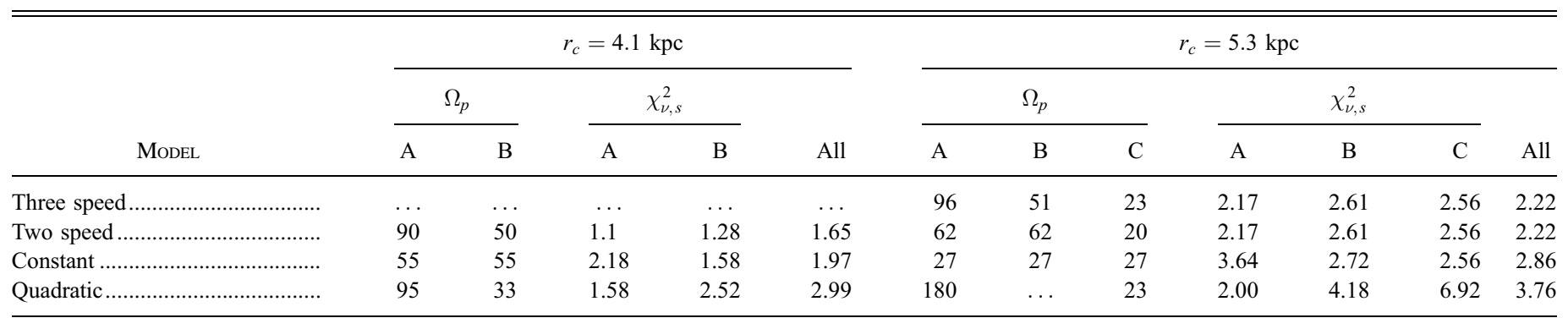

Notes.-Representation of the goodness of fit for model solutions with either $r_{c}=4.1 \mathrm{kpc}($ left $)$ or $r_{c}=5.3 \mathrm{kpc}($ right $)$ at the nominal P.A. $=170^{\circ}$. The $\chi^{2}$ estimates in the zones $0<|y|<2.3 \mathrm{kpc}, 2.3<|y|<4.0 \mathrm{kpc}$, and $4.0<|y|<5.3 \mathrm{kpc}$, labeled A, B, and C, respectively, are listed along with those for all slices, where all values are calculated as a reduced $\chi^{2}$ difference of the model reproduced to measured $\langle v\rangle_{i}$. The $\chi_{\nu, s}^{2}$ in each radial zone is normalized by the number of bins in that zone minus the number of degrees of freedom, and so represent a goodness-of-fit distinct from a $\chi_{\nu}^{2}$ over all slices with which we judge the whole solution. The multispeed solutions with $r_{c}=4.1$ and $5.3 \mathrm{kpc}$ correspond to the best-fit two and three pattern speed solutions, respectively. Pattern speed estimates in units of $\mathrm{km} \mathrm{s}^{-1} \mathrm{kpc}^{-1}$ in each zone are also listed. For quadratic solutions, the values in the first and last radial bins are indicated. 

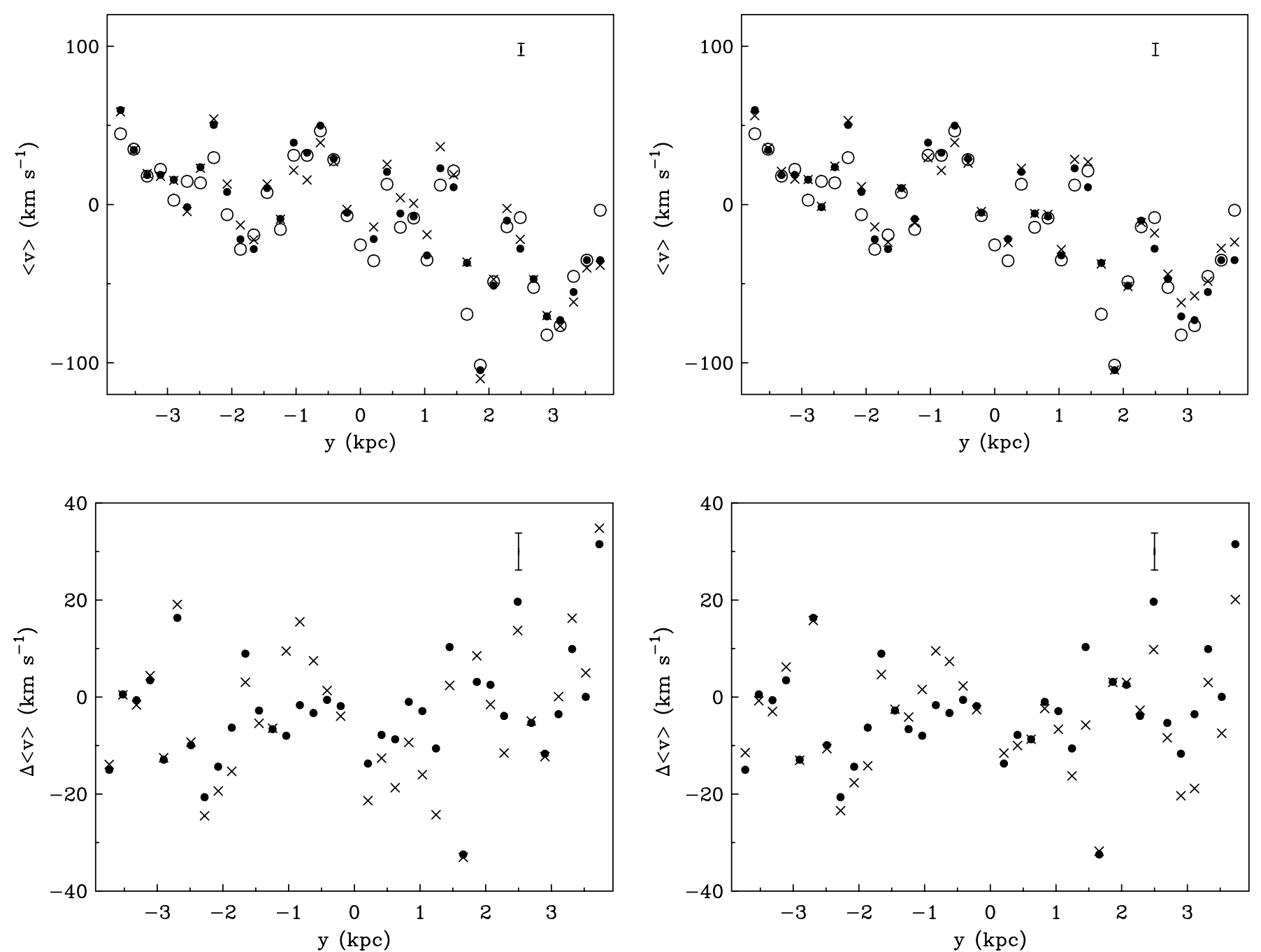

Fig. 4.-Top: Plots of solution-reproduced (dots and crosses) and actual (open circles) integrals $\langle v\rangle_{i}=b_{i} / \int \Sigma d x$ as a function of slice position $y$ at P.A. $=170^{\circ}$. The values associated with the best-fit two pattern speed solution calculated with $r_{c}=4.1 \mathrm{kpc}($ dots $)$ are plotted along with those of the order-zero polynomial solution with constant pattern speed $\Omega_{p}=55 \mathrm{~km} \mathrm{~s}^{-1} \mathrm{kpc}^{-1}$ (crosses in left panel) and the second order polynomial solution (crosses in right panel). Bottom: Plots of the residuals in $\langle v\rangle$ reproduced by the solutions considered above (left: dots for the best-fit, crosses for the constant solution; right: dots for the best-fit, crosses for the quadratic solution). The adopted global error $\sigma^{\langle v\rangle}$ is shown in the upper right in each plot. Only those slices which show a contribution from bins inward of $r_{c}=4.1 \mathrm{kpc}$ are shown. [See the electronic edition of the Journal for a color version of this figure.]

the evidence in Figures 1 and 2 that information beyond $r \simeq 4 \mathrm{kpc}$ is not conducive to modeling and extraction, the determination in the third zone is fairly accurate.

This equivalence inside $r \simeq 4 \mathrm{kpc}$ to the pattern speeds measured in the solutions with $r_{c}=4.1 \mathrm{kpc}$ derives in practice through the parameterization of a transition at $r \simeq 4 \mathrm{kpc}$. This establishes an identical radial domain for the inner speeds in the solutions with $r_{c}=5.3$ and $4.1 \mathrm{kpc}$. The transition $r_{t .1} \sim 2.3 \mathrm{kpc}$ in the three-speed solution as such yields the greatest similarity to the best-fit two-speed solution with $r_{c}=4.1 \mathrm{kpc}$.

Moreover, according to equation (2), rigorous measurement inside $4 \mathrm{kpc}$ in principle also owes to accurate measurement for the pattern speed in the zone $4 \lesssim r \lesssim 5 \mathrm{kpc}$. As inferred above, the measurement $\Omega_{p, 3}=20 \mathrm{~km} \mathrm{~s}^{-1} \mathrm{kpc}^{-1}$ is in fact lower than all measurements interior. Presumably, it is the value in this zone that contributes to the measurement of the rather low TW value $38 \mathrm{~km} \mathrm{~s}^{-1} \mathrm{kpc}^{-1}$ (Zimmer et al. 2004).

Even if the measurement for a distinct speed in the zone $4 \lesssim$ $r \lesssim 5 \mathrm{kpc}$ is a good description of the pattern there, since all $\chi^{2}$ are lower in solutions with $r_{c}=4.1 \mathrm{kpc}$ than with $r_{c}=5.3 \mathrm{kpc}$, we take this as an indication that calculating bins in the zone $4.1<r<5.3 \mathrm{kpc}$ without regularization does not reintroduce noise into solutions. Consequently, solutions with $r_{c}=4.1 \mathrm{kpc}$ should yield the more accurate description for structure in the zone $r \lesssim 4 \mathrm{kpc}$. This analysis therefore at best indicates that within $r_{c}=4.1 \mathrm{kpc}$ the data at the nominal P.A. are well fit by two pattern speeds. In addition, however, it seems possible to extend the multispeed model's estimate for $\Omega_{p}(r)$ to $5.3 \mathrm{kpc}$ without loss of validity, and this appears to be a good approximation to the pattern speeds of the structure across this zone.

Future high-resolution CARMA observations of M51 should enable the TWR measurements inside $r \lesssim 4 \mathrm{kpc}$ to be more clearly distinguished, especially at the innermost radial bins. Presently, however, it is nevertheless clear that with the radial calculation at P.A. $=170^{\circ}$ we measure a pattern speed for the bright spiral structure in the zone $r \lesssim 4 \mathrm{kpc}$ higher than the global $\sim 38 \mathrm{~km} \mathrm{~s}^{-1}$ $\mathrm{kpc}^{-1}$ found by Zimmer et al. (2004) also at P.A. $=170^{\circ}$. Interestingly, our measurement of a higher inner speed resembles the lower bound on such a pattern available with the TW calculation, $\Omega_{p} \geq 88 \mathrm{~km} \mathrm{~s}^{-1} \mathrm{kpc}^{-1}$ (Zimmer et al. 2004).

\subsection{Dependence on P.A.}

We expect the rather large P.A. uncertainty $\delta_{\text {P.A. }}= \pm 5^{\circ}$ to introduce significant variation in the values measured at the nominal 


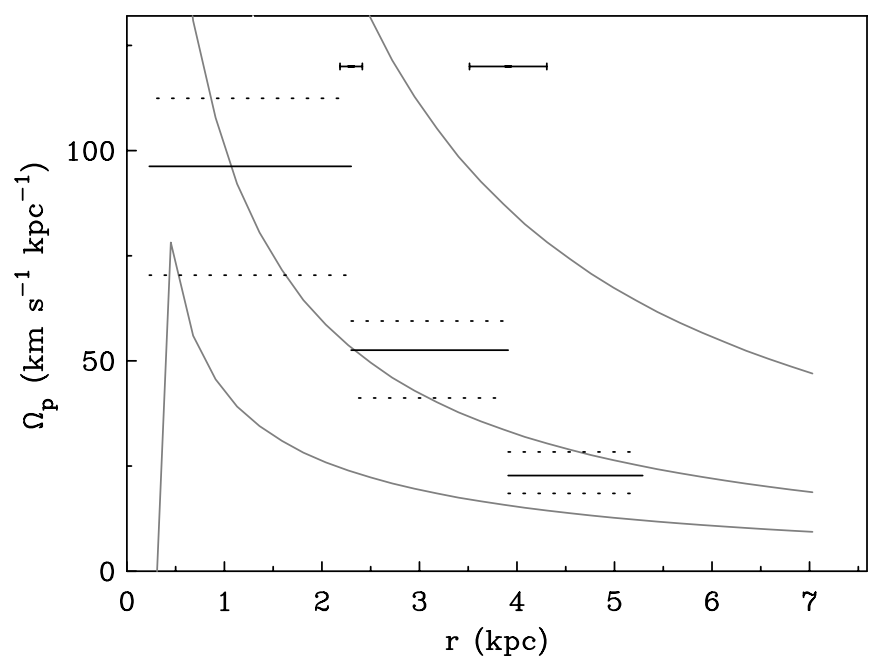

FIG. 5.-Best-fit regularized solution with $r_{c}=5.3 \mathrm{kpc}$ for P.A. $=170_{-5 \circ}^{\circ}$. For this solution, bins exterior to $r=5.3 \mathrm{kpc}$ (not shown) have been calculated without regularization. Dashed lines represent the difference from solutions derived with a three pattern speed model at P.A. $=165^{\circ}$ and $175^{\circ}$. Horizontal error bars represent the dispersion in $r_{t, 1}$ and $r_{t, 2}$ from P.A. to P.A. The values in the zone of the bright spiral structure correspond to $\Omega_{p, 1}=96_{+16}^{-26} \mathrm{~km} \mathrm{~s}^{-1} \mathrm{kpc}^{-1}$ out to $r_{t, 1}=2.3 \pm 0.1 \mathrm{kpc}, \Omega_{p, 2}=51_{-11}^{+7} \mathrm{~km} \mathrm{~s}^{-1} \mathrm{kpc}^{-1}$ out to $r_{t, 2}=3.9 \pm 0.4 \mathrm{kpc}$ and $\Omega_{p, 3}=23_{+6}^{-7} \mathrm{~km} \mathrm{~s}^{-1} \mathrm{kpc}^{-1}$ out to $r_{c}$. Curves for $\Omega, \Omega \pm \kappa / 2$, and $\Omega-\kappa / 4$ (see $\S 4.5$ ) are shown in gray. [See the electronic edition of the Journal for a color version of this figure.]

P.A.; in the previous section, we used this to define the error in the measurement of the best-fit parameters for P.A. $=170^{\circ}$. But TWR solutions from P.A. $=165^{\circ}$ and $175^{\circ}$ themselves additionally indicate a departure from the parameterization characteristic of the lowest $\chi_{\nu}^{2}$ solution measured at $170^{\circ}$.

When we identify the best-fit solutions strictly by their $\chi_{\nu}^{2}$ over all slices at each P.A. - rather than restrict our consideration at P.A. $=165^{\circ}$ and $175^{\circ}$ to pattern speed solutions optimal at P.A. $=170^{\circ}-$ we find that the values and domains of the best-fit pattern speeds vary from P.A. to P.A. Figure 6 shows the best-fit solutions at the three P.A.s, the values and $\chi_{\nu}^{2}$ for which are given in Table 3. There, solutions with $r_{c}=5.3 \mathrm{kpc}$ at P.A. $=165^{\circ}$ and $175^{\circ}$, unlike at P.A. $=170^{\circ}$, measure at most two distinct pattern speeds. More notably, although the best-fit solution with $r_{c}=4.1 \mathrm{kpc}$ at $165^{\circ}$ measures two pattern speeds, at $175^{\circ}$ no unique pattern speed is measured inside $r \lesssim 2 \mathrm{kpc}$.

Model comparisons based on $\chi_{\nu}^{2}$ over all slices and the separated $\chi_{\nu, S}^{2}$ diagnostic (Table 3 ) demonstrate the degree to which these best-fit solutions differ from those at the nominal P.A. At $165^{\circ}$, for instance, the $\chi_{\nu}^{2}$ for solutions with $r_{c}=5.3 \mathrm{kpc}$ suggest that three pattern speeds are nearly indistinguishable from the best-fit solution. The $\chi_{\nu, S}^{2}$ confirms that the third zone in solutions with $r_{c}=5.3 \mathrm{kpc}$ is fit equally as well by a third pattern speed as by the second speed in Figure 6 . (Inside $r \sim 4 \mathrm{kpc}$, the $\chi^{2}$ [and speeds] of the three- and two-speed solutions are nearly identical.) Furthermore, where $r_{c}=4.1 \mathrm{kpc}$ two pattern speeds fit the data significantly better than a single, constant pattern speed.

At P.A. $=175^{\circ}$, too, judged overall by the $\chi_{\nu}^{2}$, three (two) distinct pattern speeds seem to fit nearly as well as the best-fit solution with $r_{c}=5.3 \mathrm{kpc}(4.1 \mathrm{kpc})$. From the $\chi_{\nu, s}^{2}$ it is apparent that for $r_{c}=5.3 \mathrm{kpc}$ two pattern speeds inside $r \simeq 4 \mathrm{kpc}$ fit the inner two zones significantly better than the single pattern speed shown in Figure 6. However, the $\chi_{\nu, s}^{2}$ in the third zone of this triple pattern speed solution is rather high; the small $11 \mathrm{~km} \mathrm{~s}^{-1} \mathrm{kpc}^{-1}$ difference in the speeds measured inside $r \sim 4 \mathrm{kpc}$ therefore seems available only at the expense of accuracy in third pattern speed.

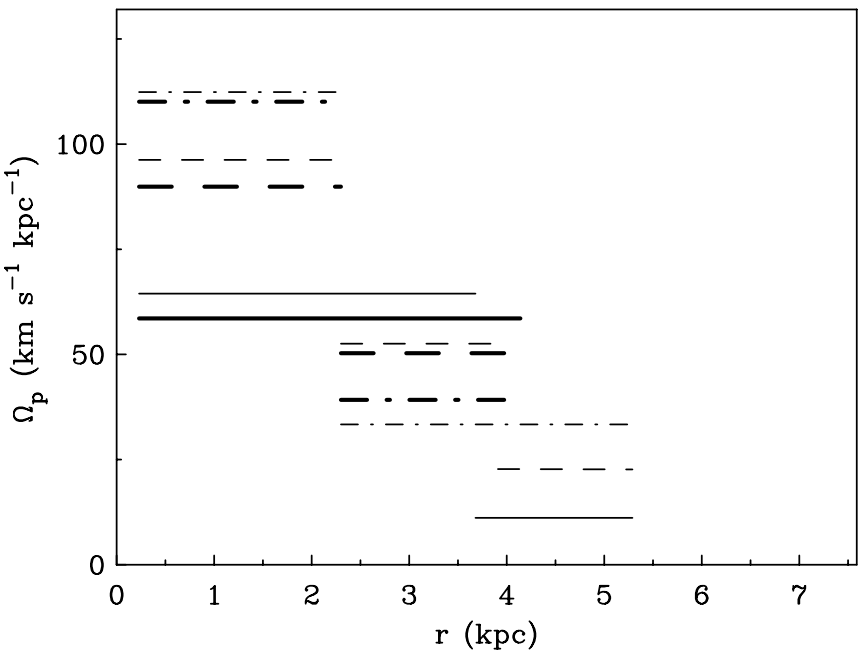

FIG. 6.-Best-fit regularized solutions at three P.A.s (P.A. $=165^{\circ}, 170^{\circ}$ and $175^{\circ}$ ). Solutions calculated with both $r_{c}=4.1$ and $5.3 \mathrm{kpc}$ are plotted, with the latter shown in thinner line. In these solutions, bins exterior to $r_{c}$ (not shown) are calculated without regularization. The solutions from P.A. $=165^{\circ}, 170^{\circ}$, and $175^{\circ}$ are shown by dash-dotted, dashed, and solid lines, respectively. Values and domains of the pattern speeds in solutions at each P.A. are given in Table 3. [See the electronic edition of the Journal for a color version of this figure.]

Furthermore, for $r_{c}=4.1 \mathrm{kpc}$ the distinction between the two pattern speeds measured inside $r \simeq 4 \mathrm{kpc}$ weakens; the two in this case are nearly identical to the constant value (such that the $\chi_{\nu}^{2}$ [and $\chi_{\nu, s}^{2}$ ] of both solutions are comparable). Overall, then, Table 3 supports a conclusion that the data at $175^{\circ}$ are at best consistent with a single constant pattern speed inside $r \sim 4 \mathrm{kpc}$.

This apparent preference for the measurement of a constant pattern speed at P.A. $=175^{\circ}$ may suggest a phenomenological difference in the projection of asymmetries (both intensity and velocity) from that in the $170^{\circ}$ case. For example, at P.A. $=$ $175^{\circ}$, and also at a higher P.A. $=180^{\circ},\langle v\rangle$ measurements in slices $|y|<2.3 \mathrm{kpc}$ are about $\sim 50 \%$ smaller than at P.A. $=170^{\circ}$, a significant difference given the flux error for these slices. While this is consistent with expectations for the large change in TW integrals introduced by a change in the P.A. (e.g., from a nominal $\delta_{\text {P.A. }}=0^{\circ}$; Debattista 2003), the smaller projected streaming velocities in this case may be more difficult to extract with the TWR calculation, and lacking strong signatures, reproducing the higher pattern speed measured at $165^{\circ}$ and $170^{\circ}$ may therefore be improbable at $175^{\circ}$. In effect, the single measured speed inside $r \simeq 4 \mathrm{kpc}$ at P.A. $=175^{\circ}$ may therefore reasonably describe two pattern speeds with significant error in each; this speed is nearly consistent with the solution plotted in Figure 3 with the errors defined by the P.A. uncertainty.

Critically, however, the data admit both P.A. $=170^{\circ}$ and P.A. $=$ $175^{\circ}$, and according to the findings of Shetty et al. (2007) addressed in $\S 4.6$, the latter may be arguably more valid at the inner radii than our chosen $170^{\circ}$. In this case, if, as might be indicated by the analysis of Shetty et al. (2007) the P.A. does not reach the assumed $170^{\circ}$ until $r \simeq 3 \mathrm{kpc}$, rather than measuring a distinct pattern speed, $\Omega_{p, 1}$ could thus be interpreted as simply identifying the region in the disk where the assumed P.A. is inappropriate. Furthermore, by the same token as above, the large (by comparison) $\langle v\rangle$ at $170^{\circ}$ may themselves reflect a misrepresentation of velocities in projection from the $175^{\circ}$ case. Consequently, if choosing $170^{\circ}$ introduces streaming motions that are unreal, the two pattern speeds measured in the best-fit solution at $170^{\circ}$ could just as persuasively reflect a large P.A. error introduced into the measure of a single constant pattern speed. 
TABLE 3

$\chi^{2}$ Model Comparisons

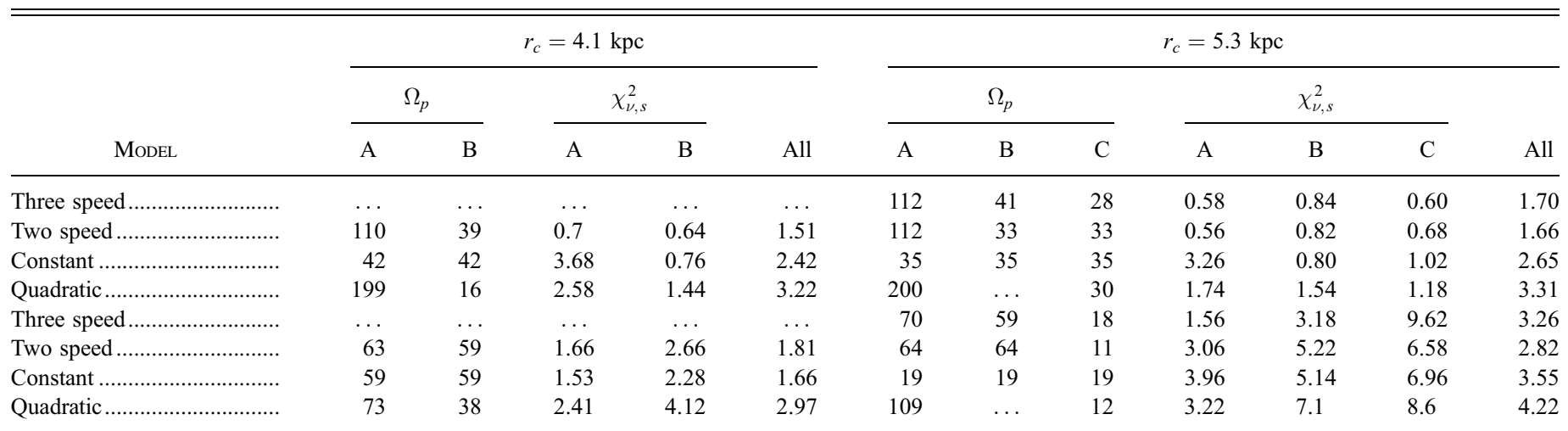

Notes.-Representation of the goodness of fit given by $\chi_{\nu, s}^{2}$, as in Table 2 , for model solutions at P.A. $=165^{\circ}$ and $175^{\circ}$ calculated with $r_{c}=4.1 \mathrm{kpc}($ left $)$ and $r_{c}=$ $5.3 \mathrm{kpc}\left(\right.$ right). Here, the zones $0<|y|<r_{t, 1}, r_{t, 1}<|y|<r_{t, 2}$, and $r_{t, 2}<|y|<5.3 \mathrm{kpc}$ are labeled A, B, and C, respectively, with transition radii as identified in the lowest $\chi^{2}$ two (three) pattern speed solution calculated with $r_{c}=4.1 \mathrm{kpc}(5.3 \mathrm{kpc})$. At $165^{\circ}$, these transitions occur at $r_{t, 1}=r_{t}=2.3 \mathrm{kpc}$, where $r_{c}=4.1 \mathrm{kpc}$ and $r_{t, 1}=2.3 \mathrm{kpc}$ and $r_{t, 2}=4.4 \mathrm{kpc}$, where $r_{c}=5.3 \mathrm{kpc}$. At $175^{\circ}, r_{t, 1}=r_{t}=2.3 \mathrm{kpc}$, where $r_{c}=4.1 \mathrm{kpc}$, and $r_{t, 1}=2.3 \mathrm{kpc}$ and $r_{t, 2}=3.7 \mathrm{kpc}$, where $r_{c}=5.3 \mathrm{kpc}$. Pattern speed estimates in units of $\mathrm{km} \mathrm{s}^{-1} \mathrm{kpc}^{-1}$ in each zone are also listed. For quadratic solutions, the values in the first and last radial bins are indicated.

Since solutions at the two P.A.s indicate quite independent radial behaviors, we include here an estimate which may be more appropriate for a nominal P.A. $=175^{\circ}$. Figure 7 plots solutions with $r_{c}=4.1 \mathrm{kpc}$ and $r_{c}=5.3 \mathrm{kpc}$ for P.A. $=175^{\circ} \pm 5^{\circ}$, where we have fixed the functional form of solutions at P.A. $=170^{\circ}$ and $180^{\circ}$ to that of the best-fit $175^{\circ}$ solution. (These solutions are also the best-fitting at P.A. $=180^{\circ}$; for $170^{\circ}$ the multipattern speed solutions in the previous section are otherwise best). Note that the values inside $r \simeq 4 \mathrm{kpc}$ are only slightly modified with the inclusion of the zone $4.1<r<5.3 \mathrm{kpc}$.

\subsection{The State of Current Measurements}

Although the P.A. of the disk is ambiguous, for the particular case of a single assumed P.A. $=170^{\circ}$, the majority of our analysis leads us to consider the solution in Figure 3 a fair repre-

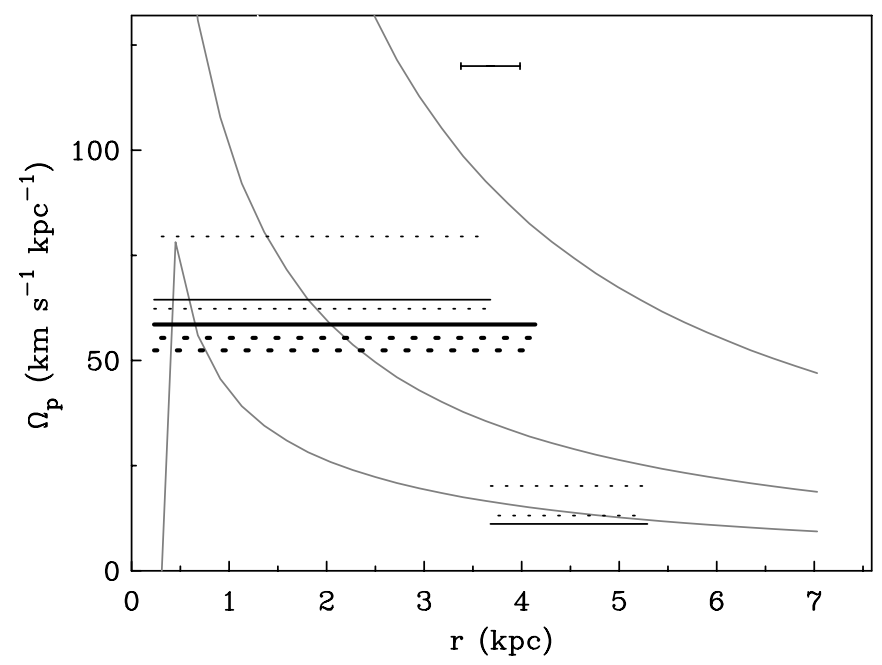

FIG. 7.-Best-fit regularized solution for P.A. $=175_{-5^{\circ}}^{\circ+50}$ and $r_{c}=4.1 \mathrm{kpc}$ $(5.3 \mathrm{kpc})$. Dashed lines represent the difference from solutions derived with a one (two) pattern speed model at P.A. $=170^{\circ}$ and $180^{\circ}$ (the best-fit functional forms at P.A. $=175^{\circ}$ ). Horizontal error bars represent the dispersion in $r_{t}$ calculated in solutions with $r_{c}=5.3 \mathrm{kpc}$ from P.A. to P.A. Where $r_{c}=4.1 \mathrm{kpc}$, the value in the zone of the bright spiral structure corresponds to $\Omega_{p, 1}=59_{-3}^{-7} \mathrm{~km} \mathrm{~s}^{-1} \mathrm{kpc}^{-1}$ out to $r_{c}=4.1 \mathrm{kpc}$ and where $r_{c}=5.3 \mathrm{kpc}$ (thinner line), $\Omega_{p, 1}=64_{+15}^{-2} \mathrm{~km} \mathrm{~s}^{-1} \mathrm{kpc}^{-1}$ out to $r_{t}=3.7 \pm 0.2$ and $\Omega_{p, 2}=11_{+9}^{+2} \mathrm{~km} \mathrm{~s}^{-1} \mathrm{kpc}^{-1}$ out to $r_{c}=5.3 \mathrm{kpc}$. Curves for $\Omega, \Omega \pm \kappa / 2$, and $\Omega-\kappa / 4$ (see $\S 4.5$ ) are shown in gray. [See the electronic edition of the Journal for a color version of this figure.] sentation of the (isolated) inner disk. As stated previously, the errors represent P.A. uncertainty introduced to the parameters of the best-fit solution derived at the nominal P.A. with a two pattern speed model. This uncertainty $\delta_{\mathrm{P} . \mathrm{A} .}= \pm 5^{\circ}$ defines $22 \%$ and $16 \%$ error on the pattern speed estimates $\Omega_{p, 1}$ and $\Omega_{p, 2}$, respectively, and $14 \%$ error in the transition $r_{t}$, all reasonable with regard to the standard set by the study of Debattista (2003).

According to the study of Meidt et al. (2008) part of this error can be expected to have originated with limitations in determining the location of the transition between the two patterns (assuming they exist), as a result of the finite radial bin width. In addition, for the inner pattern speed additional uncertainty may arise given the disparity between the inner extent of the solution and that of the true, dominant two-armed pattern, which in the surface brightness terminates at the ringlike structure at $r \sim 0.6 \mathrm{kpc}$. If structure inside $r \simeq 1 \mathrm{kpc}$, perhaps like that identified in the near-IR by Zaritsky et al. (1993) contributes to the calculation with a unique pattern speed in this zone, our measurement $\Omega_{p, 1}$ would represent a combination of this value with that for structure out to $r \sim 2 \mathrm{kpc}$. (Note, too, in this case, $\Omega_{p, 1}$ would also misestimate the true pattern speed between $0.6 \lesssim r \lesssim 2.0 \mathrm{kpc}$.) Unfortunately, identifying whether or not an additional, unique pattern exists inside $r \sim 1.0 \mathrm{kpc}$, or even establishing an innermost extent for the measure $\Omega_{p, 1}$ is currently beyond our capability; the total degrees of freedom for even the lowest order polynomial exceed the number of available bins in the innermost radial zone.

Presently, the pattern speeds in the best-fit solution at P.A. = $170^{\circ}$ in general tend to be arranged adjacent to the angular rotation curve (or perhaps even along the curve $\Omega-\kappa / 4$; see Fig. 3 or Fig. 5 showing rotation curves established in $\S 4.5$ ), much as if identifying a propensity toward a material pattern description. Rather than furnish a description for arms that are material and winding, however, we note that the very alignment of multiple pattern speeds with the disk angular rotation may relate to an underlying mechanism governing the existence and maintenance of structure in the disk. In one interpretation, the succession of corotation radii implied by the best-fit solution might be an indication of resonance overlap, as discussed inconclusively in $\S 4.5$. Associated with mode-coupling, this would allow quasi-static spiral structure to be maintained over a large portion of the disk (Sygnet et al. 1988) while transporting energy and angular momentum outward. 
Our TWR solutions furthermore seem unlike what might be expected for transient density waves, with description deriving from the propagation of tidal perturbations studied by Salo \& Laurikainen 2000b. For example, although their range of applicability seems limited to the innermost radii (but taken as an approximation to the best-fit multispeed solutions) the bin values in the current set of quadratic solutions are much closer to the angular rotation of the disk than $\Omega-\kappa / 2$, near which much of the $m=2$ structure in the models of Salo \& Laurikainen (2000b) achieves its greatest amplitude.

In order to best establish the extent to which the TWR solution in Figure 3 is truly a valid description of the bright spiral structure, in the section immediately following, and in $\S \S 4.6$ to 4.7 , we relate the radial dependence exhibited by the solution to observed morphological and kinematic structure. The inner disk of M51 has been suggested to sustain radial variation in the P.A. (Shetty et al. 2007) and an additional $m=3$ mode (Henry et al. 2003), both of which undeniably challenge the authenticity of the TWR solutions, and so we address the possible influence of each of these in turn.

\subsection{Possible Complimentary Evidence for Multiple Pattern Speeds and Indications of Mode Coupling}

Although perhaps unexpected, the identification of a transition between two pattern speeds in the inner disk seems supported by independent studies of the bright spiral structure. At least two sections best fit with slightly different pitch angles $i_{p}$ have been identified in both spiral arms, possibly the signature of two or more distinct pattern speeds. Notably, the anisotropic wavelet approach of Patrikeev et al. (2006) shows evidence for extreme departures from the conventionally adopted value $i_{p}=21^{\circ}$. The maximum occurring nearly symmetrically in both arms at $r \sim$ 2 kpc (see Figs. 6, 7a, and $8 a$ in Patrikeev et al. 2006) - very near the transition $r_{t}$ identified in our best-fit solution at P.A. $=170^{\circ}-$ is especially compelling since it may indicate more than a simple departure from a logarithmic dependence. The transition $r_{t, 2}$ in Figure 5 also occurs near a maximum in $i_{p}$. (To be sure, the other extrema in $i_{p}$ imply no such correlation.) This analysis is largely consistent with the findings of Henry et al. (2003) covering radii $r \lesssim 4 \mathrm{kpc}$ which identify three arm sections each with a unique $i_{p}$.

Of course, a systematic error in deprojection, such as due to an incorrectly adopted P.A. or inclination angle (or a radially varying P.A., as considered in $\S 4.6$ ) could very well alone produce the effect measured by Patrikeev et al. (2006) (where P.A. $=170^{\circ}$ ). A firm conclusion might therefore require a better understanding of how a change in pitch angle at a given radius relates to a change in pattern speed, for instance (assuming that the spirals are indeed logarithmic).

The transition between two patterns inside $r \simeq 4 \mathrm{kpc}$ indicated in our solution also seems significant given that it coincides with features in the zeroth moment map's Fourier decomposition (Fig. 2); as at $r \sim 4 \mathrm{kpc}$, the power in the $m=2$ mode is characterized by a decline at $r \sim 2 \mathrm{kpc}$ possibly marking the termination of a distinct structure. (The same can be inferred at $4 \mathrm{kpc}$ which coincides with the transition $r_{t, 2}$ in the three pattern speed solution.)

Perhaps more compellingly, the transitions parameterized in our solutions at $170^{\circ}$ also appear to coincide with resonances, as illustrated in Figures 3 and 5. This, of course, would seem to depend largely on the assumed rotation curve. As first demonstrated by Tully (1974) streaming motions appear nonnegligibly in the rotation curve of M51, making the true circular velocity difficult to constrain. As more recently cataloged by Shetty et al. (2007) all other kinematic parameters are likewise susceptible to such errors, and so rotation curves generated with them are prone to inaccuracy. To reduce the impact of streaming motions (and perhaps other systematic errors) on our resonance identifications, we fit our own ROTCUR-derived rotation curve with the commonly used approximation (e.g., by Faber \& Gallagher 1979)

$$
V_{\text {rot }}(r)=\frac{V_{\max }\left(r / r_{\max }\right)}{\left[1 / 3+2 / 3\left(r / r_{\max }\right)^{n}\right]^{3 / 2 n}},
$$

which yields a smoothed curve for $\Omega$. In this expression, $V_{\max }$ is the maximum rotational velocity, $r_{\max }$ is the location where $V_{\max }$ occurs, and $n$ determines how rapidly the curve becomes Keplerian. Alternative fits (like that used on the inner $30^{\prime \prime}$ by Aalto et al. 1999) supply similar conclusions.

With the resulting curves for $\Omega, \Omega-\kappa / 2, \Omega-\kappa / 4$, and $\Omega+$ $\kappa / 2$ plotted in Figures 3 and 5 we highlight the possible locations for the corotation, inner Lindblad, inner 4:1 ultraharmonic, and outer Lindblad resonances (or CR, ILR, UHR, and OLR) for each measured pattern speed. Immediately we notice that both pattern speeds in the solution with $r_{c}=4.1 \mathrm{kpc}$ end at their CR within the uncertainties. This circumstance is consistent with an early prediction for where spirals terminate (e.g., Lin 1970), which later yielded to findings that spirals can extend as far as OLR, if sometimes faintly (see Zhang \& Buta 2007 and references therein).

In addition, the transition between the two pattern speeds appears to occur at a resonance overlap. As demonstrated in Figure 3, the CR of $\Omega_{p, 1}$ overlaps the UHR of the pattern with $\Omega_{p, 2}$. Such coincidences have been identified in barred spiral simulations of Rautiainen \& Salo (1999) and Debattista et al. (2006). As the former investigate, this overlap at resonance may be characteristic of nonlinear mode coupling (e.g., Tagger et al. 1987 and Sygnet et al. 1988), whereby energy and angular momentum are transferred between the modes. But in contrast to the CR-ILR overlaps studied by Masset \& Tagger (1997) which are accompanied by boosted beat modes detectable in the simulation power spectra at the overlap, they find no comparable evidence for modecoupling in the case of the CR-UHR overlap. (They suggest this overlap may nevertheless be related to a physical process.)

A CR-ILR overlap between $\Omega_{p, 1}$ and $\Omega_{p, 3}$ in the solution with $r_{c}=5.3 \mathrm{kpc}$, on the other hand, may be viable within the uncertainties. However, between $\Omega_{p, 2}$ and $\Omega_{p, 3}$ in Figure 5 a similar resonance overlap is not so clear; near the transition $r_{t, 2} \mathrm{CR}$ of $\Omega_{p, 2}$ falls between the ILR and the UHR of $\Omega_{p, 3}$.

Figure 3 also exhibits a turnover in the curve $\Omega-\kappa / 2$, suggesting that patterns with angular speeds above the maximum lack an ILR. However, given uncertainty in the rotation curve, this is difficult to constrain: while the angular frequency curves of Tully (1974) indicate that $\Omega-\kappa / 2 \sim 47 \mathrm{~km} \mathrm{~s}^{-1} \mathrm{kpc}^{-1}$ at the turnover (also reproduced in the Salo \& Laurikainen [2000b] model), we find that the maximum occurs at $\sim 75 \mathrm{~km} \mathrm{~s}^{-1} \mathrm{kpc}^{-1}$. [Our fit for $\Omega(r)$ may be slightly steep inside $\sim 1.0 \mathrm{kpc}$.] Nevertheless, it is apparent for this solution that $\Omega_{p, 1}$ lacks an ILR. This suggests that a (trailing) wave with $\Omega_{p, 1}$ can reflect from the center as a leading structure, a circumstance complimentary to the Scoville et al. (2001) HST observations of central leading waves, as pointed out by Salo \& Laurikainen (2000b).

\subsection{Effect of a Radial Variation in P.A.}

From their analysis of the $\mathrm{CO}$ and $\mathrm{H} \alpha$ kinematics, Shetty et al. (2007) find evidence for large nonzero radial flux (as measured by the mass/surface brightness-weighted radial velocity) in radial ranges that depend on the choice of P.A. From Figure 18 of 


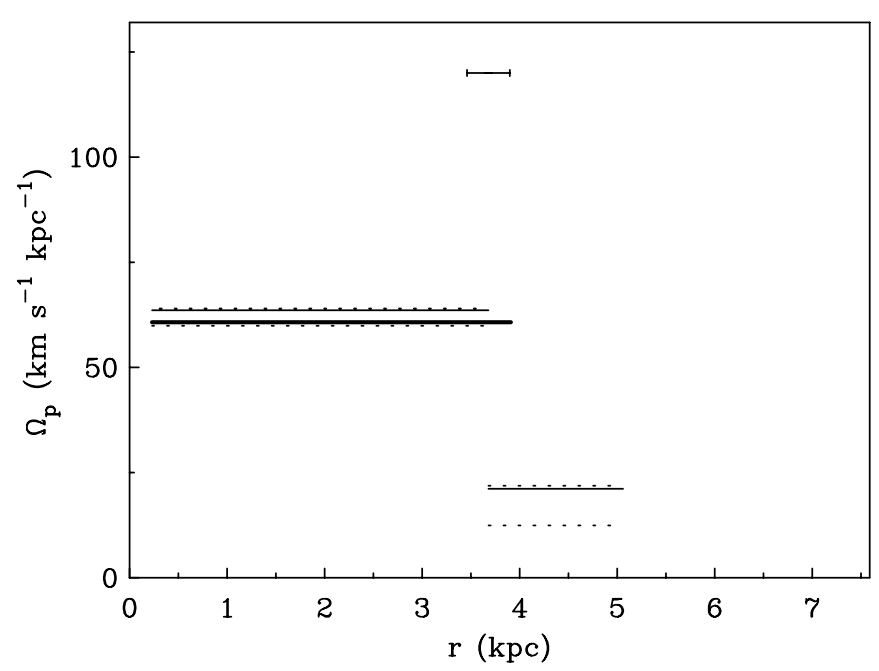

FIG. 8. - Best-fit regularized solution for the P.A. twist map with $\delta_{\text {P.A. }}= \pm 2^{\circ}$ and $r_{c}=4.1 \mathrm{kpc}(5.3 \mathrm{kpc}$ ) shown in thick (thin) line. (See text for a description.) Dashed lines represent the difference from solutions derived with a two pattern speed model best for the $\delta_{\text {P.A. }}=0^{\circ}$ case; errors for the solution with $r_{c}=4.1 \mathrm{kpc}$ are nearly coincident with these lines and have been left off for clarity. Horizontal error bars represent the dispersion in $r_{t}$ calculated in solutions with $r_{c}=5.3 \mathrm{kpc}$ Where $r_{c}=4.1 \mathrm{kpc}$, the value in the zone of the bright spiral structure corresponds to $\Omega_{p}^{\text {P.A. }}=61_{-1}^{+4} \mathrm{~km} \mathrm{~s}^{-1} \mathrm{kpc}^{-1}$ out to $r_{c}=4.1 \mathrm{kpc}$ and where $r_{c}=5.3 \mathrm{kpc}$ (thinner line), $\Omega_{p, 1}^{\text {P.A. }}=62 \pm 2 \mathrm{~km} \mathrm{~s}^{-1} \mathrm{kpc}^{-1}$ out to $r_{t}=3.8 \pm 0.5 \mathrm{kpc}$ and $\Omega_{p, 2}^{\text {P.A. }}=18 \pm 2 \mathrm{~km} \mathrm{~s}^{-1} \mathrm{kpc}^{-1}$ out to $r_{c}=5.3 \mathrm{kpc}$. For there solutions, bins exterior to $r_{c}$ (not shown) are calculated without regularization. [See the electronic edition of the Journal for a color version of this figure.]

that study, in particular, Shetty et al. (2007) speculate that mass flux could be conserved should the disk of M51 sustain a radially dependent P.A. (and/or inclination). This could be approximately achieved with P.A. $=180^{\circ}$ out to $r \sim 1.8 \mathrm{kpc}$, P.A. $=175^{\circ}$ out to $r \sim 2.8 \mathrm{kpc}$ and P.A. $=170^{\circ}$ out to $r \sim 3.7 \mathrm{kpc}$. (The inclination angle, which might also be expected to vary, is much harder to account for in the TWR calculation.)

If the P.A. does vary radially then the measurement in Figure 3 (or Fig. 7) could be affected by projection errors at certain radii. Note that a radially varying P.A. implies a warp in the inner disk (which, if real, could be due to the presence of the companion) and so the disk would also not meet the assumptions of the TWR method. Interpreted in this manner, our finding of possible multiple pattern speeds in the inner disk may be the result of such an effect.

We explore this possibility by allowing the P.A. to vary radially in the TWR quadrature according to the prescription given at the beginning of this subsection. For simplicity, we retain $i=24^{\circ}$ throughout the disk and let P.A. $=170^{\circ}$ at all radii beyond $r \sim$ $3.7 \mathrm{kpc}$. Figure 8 shows the best-fit solutions with $r_{c}=4.1 \mathrm{kpc}$. Errors represent a residual P.A. uncertainty $\delta_{\text {P.A. }}= \pm 2^{\circ}$ estimated from Figure 18 of Shetty et al. (2007).

Interestingly, the global pattern speed inside $r_{c}=4.1 \mathrm{kpc}$ $\left(\Omega_{p}^{\text {P.A. }}=62 \pm 2 \mathrm{~km} \mathrm{~s}^{-1} \mathrm{kpc}^{-1}\right)$ closely resembles the measurement at P.A. $=175^{\circ}$ (Fig. 7). The best-fit solution with $r_{c}=$ $5.3 \mathrm{kpc}\left(\Omega_{p, 1}^{\mathrm{P} . \mathrm{A}}=62 \pm 2 \mathrm{~km} \mathrm{~s}^{-1} \mathrm{kpc}^{-1}\right.$ out to $r_{t}=3.8 \pm 0.5 \mathrm{kpc}$, $\Omega_{p, 2}^{\text {P.A. }}=18 \pm 2 \mathrm{~km} \mathrm{~s}^{-1} \mathrm{kpc}^{-1}$ out to $r_{c}$ ) also resembles that at $175^{\circ}$, and here the pattern speed in the zone $4 \lesssim r \lesssim 5 \mathrm{kpc}$ does not seem to be the result of an incorrectly assumed P.A. (i.e., P.A. $=170^{\circ}$ instead of $165^{\circ}$ ); extending the twist by another $5^{\circ}$ at radii greater than $r=3.7 \mathrm{kpc}$ produces little change in the calculated solutions.

That the estimates in Figures 8 and 7 are so similar seems to suggest the twist solution is less a manifestation of the P.A. twist than an indication that the P.A. assumed here inside $r \simeq 3 \mathrm{kpc}$ is everywhere closer to $175^{\circ}$ than $170^{\circ}$. The mean P.A. of the twist is $175^{\circ}$, so it may be reasonable to infer that the twist solution predominantly reflects information nearly identically to the $175^{\circ}$ case. Solutions at $180^{\circ}$, too, are very similar to those at $175^{\circ}$, as indicated by the estimate assembled in Figure 7.

Since imposing the twist does not seem to introduce a novel character to the TWR measurement, by extension this leads us to conclude that the regularized TWR calculation is insensitive to minor radial variation in the P.A. (i.e., $\delta_{\text {P.A. }}= \pm 5^{\circ}$ over roughly $4 \mathrm{kpc}$ ), if real. However, if the disk P.A. is assertably closer to $175^{\circ}$ than $170^{\circ}$, this seems to reinforce the impression that the bright spiral structure may be best described by a single constant pattern speed.

Although compelling, we emphasize that this exercise should not be interpreted as confirmation or denial of radial variation in the P.A., nor as providing an unequivocal measure for the pattern speed of the bright spiral structure. Critically, imposing the twist tends to remove the most noticeable asymmetries in the velocity field, particularly within $60^{\prime \prime}$, very much in the manner described previously for P.A. $=175^{\circ}$. If true signatures of pattern speeds have been obscured or eliminated at this P.A., this may prevent the measurement of a distinct pattern speed inside $r \sim 2.0 \mathrm{kpc}$.

\subsection{Relation to $m=3$ Structure}

In principle, TWR measurements at either P.A. $=170^{\circ}$ or $175^{\circ}$ may reflect signatures of patterns other than those of the bright two-armed structure alone. The Fourier power spectrum of the surface brightness reveals rich structure in the disk of M51, much of it coexisting over roughly $2 \mathrm{kpc}$ in the inner disk. In this section we review the particular possibility that weak $m=3$ structure identified by Rix \& Rieke (1993) out to $\sim 2.5 \mathrm{kpc}$ contributes with a unique pattern speed to out TWR solutions.

Only if all structures in the same radial zone have identical pattern speeds will the TWR calculation accurately reflect this sole speed; given a surface brightness distribution which reflects two coincident contributions (say, from $m=2$ and $m=3$ structures) with unique time dependence (i.e., different pattern speeds), the TWR calculation is currently unequipped to constrain either one or the other. Although a generalization can be made under the assumption that both pattern speeds are constant, it is beyond the scope of this work to develop either the TW or TWR calculation appropriate to the situation.

For M51, it may be possible in the future to directly relate the velocity asymmetry from arm to arm of the bright $m=2$ spiral structure identified by Henry et al. (2003) to the presence of the $m=3$ mode, and to its pattern speed in particular. (Henry et al. 2003 have already successfully demonstrated that the presence of the $m=3$ mode induces a systematic offset in the azimuthal positions of the two main arms). This should allow us to establish the expected combination of speeds in the TWR calculation; if the implied $m=3$ pattern speed is unlike the measure $\Omega_{p, 1}$ found with P.A. $=170^{\circ}$, for example, this speed is presumably unshared by the $m=2$ structure.

\section{CONCLUSION}

In this paper we present regularized TWR solutions for the pattern speed of the bright spiral structure in the inner disk of M51, derived with velocity and intensity information from the ISM-dominant molecular component traced by high-resolution CO observations. These solutions are arrived at by isolating the inner disk from errors which evidently originate with both the quality of sampling/detection and the pattern speed-modeling in bins covering the outer, material pattern. So although our procedure prevents us from constraining the outermost pattern speed, 
calculating the outer bins without regularization in principle improves the accuracy with which the solution for the inner disk can realize the true pattern speed.

Our primary result with this implementation is the measurement inside $4 \mathrm{kpc}$ of two pattern speeds, both significantly higher, and together fitting the data better, than the constant global measure of Zimmer et al. (2004) at the nominal P.A. $=170^{\circ}$. A third, lower pattern speed, extending beyond $4 \mathrm{kpc}$ out to (at least) $5.3 \mathrm{kpc}$ is also detected, nearer the speed expected for the material pattern. Significantly, the transitions between the measured pattern speeds coincide with resonances; the two pattern speeds inside $4 \mathrm{kpc}$ both end at corotation within the uncertainties. Since it is in no way imposed by the method, this dynamically reasonable scenario tends to give us confidence as to the physical plausibility of the pattern speeds returned by the analysis.

Of course, given that a pattern speed interior to $r \simeq 2 \mathrm{kpc}$ is only weakly detected (if at all) at P.A. $=175^{\circ}$, the accuracy of the description provided by two pattern speeds may depend on whether P.A. $=170^{\circ}$ or P.A. $=175^{\circ}$ is more accurate, an uncertainty raised by Shetty et al. (2007) for instance. If the disk is best described with P.A. $=175^{\circ}$, we find evidence that a single constant pattern speed inside $4 \mathrm{kpc}$ best characterizes the bright spiral structure. Furthermore, as contemplated in $\S 4.6$, a radially varying P.A. which decreases from $180^{\circ}$ (and reaches $170^{\circ}$ near $3 \mathrm{kpc}$ ), perhaps suggested by the results of Shetty et al. (2007) also favors a single measured pattern speed interior to $4 \mathrm{kpc}$.

Again, however, while the analysis presented here cannot resolve the question as to which P.A. is more appropriate, we find meaningful, independent evidence in favor of the pattern speeds measured at $170^{\circ}$, in particular. For example, consistent with expectations of leading structure at the inner most radii (as in the observations of Scoville et al. 2001), the higher speed inside $r \simeq$ $2 \mathrm{kpc}$ lacks an ILR. In addition, attendant to our finding that both speeds interior to $4 \mathrm{kpc}$ terminate at corotation, the transition between the two roughly coincides with an inferred location of resonance overlap wherein the inner's corotation resonance and the outer's inner 4: 1 resonance align. The radial domain of the pattern speed measured at P.A. $=175^{\circ}$, in contrast, is not as clearly associated with resonance radii. Since the bright spiral structure does not appear along the minor axis near $\sim 2 \mathrm{kpc}$, the corotation resonance at this location implied by the solution at $170^{\circ}$ is unfortunately unconfirmable through inspection of radial streaming velocities under the density wave interpretation.

We also find remarkable agreement between the characteristics of the two speeds inside $4 \mathrm{kpc}$ at P.A. $=170^{\circ}$ and other evidence in the inner disk consistent with multiple pattern speeds. The transition parameterized in our best-fit solution for the inner disk coincides with significant variation in the two-armed spiral pitch angle. Since a change in the pitch angle is expected to be accompanied by a change in streaming motions, both parameters are presumably attendant to the signatures (streaming or otherwise) of the patterns.
Although the pattern speed interior to $2 \mathrm{kpc}$ in the solution at $170^{\circ}$ (or $175^{\circ}$ ) may reflect a unique contribution from the $m=3$ mode observed by Rix \& Rieke (1993) as described in $\S 4.7$, the measurements in Figure 5 (or Fig. 7) presumably directly relate to the patterns present in the disk and so (depending on the P.A.) should provide a fair description of the dynamics therein. As such, it may be possible that ensuing observations and studies better discriminate between the two seemingly disparate radial dependencies implied for the P.A.s considered here.

Even at present our TWR solutions yield interpretations with which to observationally address the question of spiral longevity. That the solutions at both $170^{\circ}$ and $175^{\circ}$ feature constant pattern speeds would imply that our solutions are indicative of longlasting spiral structure. Interestingly, at the innermost radii both qualitatively resemble the model of Salo \& Laurikainen (2000b), where, characteristic of the isolated evolution of the disk, the dominant $m=2$ component has a constant pattern speed $\sim 50 \mathrm{~km} \mathrm{~s}^{-1}$ $\mathrm{kpc}^{-1}$ out to $\sim 1.2-1.8 \mathrm{kpc}$. As for the region between $\sim 1.8$ and $4.6 \mathrm{kpc}$ in those models where interaction with the companion introduces a succession of transient structures, our solutions at both $170^{\circ}$ and $175^{\circ}$ otherwise describe at most two steady patterns in distinct radial zones.

In the immediate future, observations with higher resolution and sensitivity should afford TWR calculations with finer radial bins, thereby allowing for the parameterization of more distinct radial zones, if present. This will either confirm our solutions for $\Omega_{p}(r)$ or perhaps demonstrate that solutions describe a succession of many discrete patterns (similar to the transient structures in the Salo \& Laurikainen (2000b) models), or simply a winding, material pattern. Again, however, our multiple-speed and other quadratic solutions in general more closely follow $\Omega$ throughout the disk than $\Omega-\kappa / 2$ characteristic of $m=2$ structure in the models of Salo \& Laurikainen (2000b).

Despite the lingering ambiguity in the P.A., these TWR solutions present a new picture of the bright spiral structure of M51, one that should prompt tests of long-lived density wave theories in other nearby grand-design spirals. At the very least, this study marks a successful starting point for continued tests of the relation between multiple spiral pattern speeds in a single disk; investigations into the number and radial domains of pattern speeds and spiral winding in nearby spirals will be the subject of upcoming work.

S. E. M. acknowledges support from a NASA-funded New Mexico Space Grant Consortium Graduate Research Fellowship. We thank Heikki Salo for providing insight into some of the intricacies of the simulations in Salo \& Laurikainen (2000b). This material is based upon work supported by the National Science Foundation under grant AST 08-07032 to R. J. R. M. R. M. gratefully acknowledges the support of an STFC Senior Fellowship.

\section{REFERENCES}

Aalto, S., Huttemeister, S., Scoville, N. Z., \& Thaddeus, P. 1999, ApJ, 522, 165

Bresolin, F., Garnett, D. R., \& Kennicutt, R. C. 2004, ApJ, 615, 228

Debattista, V. P. 2003, MNRAS, 342, 1194

Debattista, V. P., Mayer, L., Carollo, C. M., Moore, B., Wadsley, J., \& Quinn, T. 2006, ApJ, 645, 209

Elmegreen, B. G., Elmegreen, D. M., \& Seiden, P. E. 1989, ApJ, 343, 602

Faber, S. M., \& Gallagher, J. S. 1979, ARA\&A, 17, 135

Garcia-Burillo, S., Combes, F., \& Gerin, M. 1993, A\&A, 274, 148

Henry, A. L., Quillen, A. C., \& Gutermuth, R. 2003, AJ, 126, 2831

Howard, S., \& Byrd, G. G. 1990, AJ, 99, 1798
Lin, C. C. 1970, in IAU Symp. 38, The Spiral Structure of Our Galaxy, ed. W. Becker \& G. I. Contopoulos (Dordrecht: Reidel), 377

Lin, C. C., \& Shu, F. H. 1964, ApJ, 140, 646

Masset, F., \& Tagger, M. 1997, A\&A, 322, 442

Meidt, S. E., Rand, R. J., Merrifield, M. R., Debattista, V. P., \& Shen, J. 2008, ApJ, 676, 899 (M08)

Merrifield, M. R., Rand, R. J., \& Meidt, S. E. 2006, MNRAS, 366, L17

Miller, K. 1970, SIAM J. Math. Analysis, 1, 52

Patrikeev, I., Fletcher, A., Stepanov, R. Beck, R., Berkhuijsen, E. M., Frick, P., \& Horellou, C. 2006, A\&A, 458, 441

Rand, R. J. 1993, ApJ, 410, 68 
Rand, R. J., \& Wallin, J. F. 2004, ApJ, 614, 142

Rautiainen, P., \& Salo, H. 1999, A\&A, 348, 737

Rix, H. W., \& Rieke, M. J. 1993, ApJ, 418, 123

Salo, H., \& Laurikainen, E. 2000a, MNRAS, 319, 377 2000b, MNRAS, 319, 393

Scoville, N. Z., Polletta, M., Ewald, S., Stolovy, S. R., Thompson, R., \& Rieke, M. 2001, AJ, 122, 3017

Shetty, R., Vogel, S. N., Ostriker, E. C., \& Teuben, P. J. 2007, ApJ, 665, 1138 Sygnet, J. F., Tagger, M., Athanassoula, E., \& Pellat, R. 1988, MNRAS, 232, 733

Tagger, M., Sygnet, J. F., Athanassoula, E., \& Pellat, R. 1987, ApJ, 318, L43

Tikhonov, A. N., \& Arsenin, V. Y. 1997, Solutions of Ill-Posed Problems (New York: Wiley)
Toomre, A. 1981, in The Structure and Evolution of Normal Galaxies, ed. S. M. Fall \& D. Lynden-Bell (London: Cambridge Univ. Press), 111

Toomre, A., \& Toomre, J. 1972, ApJ, 178, 623

Tully, R. B. 1974, ApJS, 27, 449

Tremaine, S., \& Weinberg, M. D. 1984, ApJ, 282, L5

Vogel, S. N., Rand, R. J., Gruendel, R. A., \& Teuben, P. J. 1993, PASP, 105, 666 Zaritsky, D., Rix, H.-W., \& Rieke, M. 1993, Nature, 364, 313

Zhang, Z., \& Buta, R. J. 2007, AJ, 133, 2584

Zimmer, P., Rand, R. J., \& McGraw, J. T. 2004, ApJ, 607, 285 